\title{
Synthetic Studies Toward (-)-FR901483 Using a Conjugate Allylation to Install the C-1 Quaternary Carbon
}

\author{
Dimitar B. Gotchev and Daniel L. Comins ${ }^{\star}$ \\ Department of Chemistry, North Carolina State University, Raleigh, North Carolina 27695-8204
}

\begin{abstract}
Two approaches to the aza-tricyclo dodecane skeleton of (-)-FR901483 are reported. Both routes utilized a Grignard addition to an $\mathrm{N}$-acylpyridinium salt to establish the absolute stereochemistry at C-6 and a highly diastereoselective conjugate allylation reaction to form the quaternary center at C-1 of the natural product in excellent yield. Although the desired polysubstituted piperidine intermediates were prepared regio- and stereoselectively, the construction of the C-8/C-9 bond connectivity could not be achieved. All attempts at a pinacol cyclization or an intramolecular 6-exotet epoxide opening were unsuccessful due to unfavorable $\mathrm{A}^{1,3}$ strain inherent in the molecule.
\end{abstract}

\section{Introduction}

The immunosuppressive natural products cyclosporine A and FK 506 are leading therapeutic agents in the organ transplant field. ${ }^{1,2}$ The mechanism of action of both cyclosporin A and FK 506 is the inhibition of calcineurin, a calcium-dependent serine/threonine phosphatase.

Calcineurin, in turn, leads to the inhibition of interleukin-2 (IL-2) production, which is a signal molecule that induces cytotoxic T-cells. In high doses these two compounds exhibit serious side effects such as neurotoxicity and adverse reactions in diabetic patients. ${ }^{3}$ Because of this, the search for less toxic immunosuppressants with a different mechanism of action has emerged. These efforts led to the isolation of (-)-FR901483 (1) from the fermentation broth of the fungal strain Cladobotrium sp. No. 11231 by the Fujisawa research group, and it was shown to significantly prolong the graft survival time in the rat skin allograft model. ${ }^{4}$ More importantly, it was suggested that the mechanism of action is quite different as compared to cyclosporin A and FK 506, namely (-)-FR901483 inhibits the adenylosuccinate synthetase and/or adenylosuccinate lyase enzymes in the purine biosynthesis. Because of the high degree of bioactivity and the unprecedented azatricyclic structure of (-)-FR901483, it has become a popular target of synthetic interest. 5

In the previous syntheses of FR901483 (1), the pyrrolidine portion of the aza-tricyclo dodecane nucleus was installed utilizing a nitrone cycloaddition/hydrogenolysis, $5 \mathrm{a}$ an oxidative spiroannulation, $5 \mathrm{~b}-\mathrm{c}$ a Diels-Alder cycloaddition/aldol cyclization, $5 \mathrm{~d}$ a Michael addition/ hydrogenolysis, $5 \mathrm{e}$ or an aza-Cope rearrangement/Mannich cyclization tactic. ${ }^{5 \mathrm{f}}$ Most of the approaches to the bicyclic [3.3.1] ring system of $\mathbf{1}$ utilized an intramolecular aldol cyclization reaction. ${ }^{5 \mathrm{a}-\mathrm{e}}$ Alternatively, the bicyclic [3.3.1]-ring system can be installed through an intermediate bridgehead imminium ion, followed by an intramolecular aza-Cope rearrangement $5 \mathrm{f}$ or an intermolecular alkylation. ${ }^{5 \mathrm{~h}}$ The [3.3.1]-nonane nucleus was also successfully introduced utilizing an intramolecular carboradical cyclization. $5 \mathrm{~g}, 5 \mathrm{~m}$ Our approach to the synthesis of $\mathbf{1}$ significantly differs from the previous synthetic endeavors in both strategy and execution. Retrosynthetically, we envisioned the target $\mathbf{1}$ could be derived 
via a reductive amination of ketone $\mathbf{2}$, followed by phosphorylation at C-9 (Scheme 1). Ketone $\mathbf{2}$ in turn could be synthesized from diol $\mathbf{3}$ by the selective formation and reduction of a tertiary carbocation at C-8. Unlike the other approaches to 1, we chose to exploit a Mitsunobu-type cyclization to construct the pyrrolidine portion of the aza-tricyclo dodecane nucleus. This would then be followed by an oxidative cleavage of the double bond to give compound $\mathbf{2}$. Diol 3, in turn, could be obtained by performing an intramolecular pinacol cyclization of ketoaldehyde 4 , which could be accessed by a conjugate addition of an allylsilane or allyltin reagent such as $\mathbf{6}$ to dihydropyridone $\mathbf{5}$. Vinylogous amide $\mathbf{5}$ can be accessed via a tandem conjugate addition/oxidation in dihydropyridone 7 . Finally, Grignard addition to the chiral 1acylpyridinium salt $\mathbf{8}$ is expected to furnish enantiopure $\mathbf{7}$.

\section{Results and Discussion}

Our approach to the synthesis of (-)-FR901483 (1) commenced with Grignard addition to the chiral 1-acylpyridinium salt prepared from 3-triisopropylsilyl-4-methoxypyridine $(\mathbf{8})$ and the chloroformate of (+)-trans-2-( $\alpha)$-cumylcyclohexanol (TCC), thus establishing the absolute stereochemistry at the C-6 position of the resulting dihydropyridone 9 (90\%, $d r$ 95:5, Scheme 2). ${ }^{6,7}$ The resulting dihydropyridone 9 was obtained as a single diastereomer after recrystallization from ethyl acetate/hexanes. A one-pot reaction with sodium methoxide in methanol to remove the chiral auxiliary, followed by protodesilylation with $10 \%$ aqueous $\mathrm{HCl}$, yielded an intermediate vinylogous amide, ${ }^{8}$ which in turn was reacylated by exposure to $n$ BuLi and phenyl chloroformate to give dihydropyridone $\mathbf{7}$ as a single enantiomer.

After establishing a practical route to the optically active dihydropyridone 7, we turned our attention to the synthesis of racemic intermediates for initial studies of the feasibility of the proposed pinacol coupling and reductive amination reactions (Scheme 1). Use of the enantiopure $\mathbf{7}$ would lead to the asymmetric synthesis of $\mathbf{1}$, since once the absolute stereochemistry at C-6 is established, the rest of the stereocenters will be introduced relative to the one at C-6 in the same manner as in the racemic route. The addition of $p$ methoxybenzylmagnesium chloride to the 1-acylpyridinium salt, prepared from 4methoxypyridine (10) and phenyl chloroformate, provided dihydropyridone $\mathbf{1 1}$ in $77 \%$ yield (Scheme 3). ${ }^{6}$ Copper-mediated addition of the Grignard reagent derived from 2-(2bromoethyl)-1,3-dioxolane to compound $\mathbf{1 1}$ in the presence of trimethylsilylchloride afforded the desired silyl enol ether 12, which was then subjected to catalytic $\mathrm{Pd}(\mathrm{OAc})_{2}$ oxidative rearrangement conditions to give vinylogous amide $\mathbf{1 3}$ in $70 \%$ yield (2 steps). ${ }^{9}$ Reaction of dihydropyridone 13 with $\mathrm{Pb}(\mathrm{OAc})_{4}$ resulted in $\mathrm{C}-3$ acetoxylation to give acetate 15a as the major diastereomer $(75 \%, d r 15: 1) .{ }^{10}$ The resulting trans stereochemistry can be explained by stereoelectronic control: in order to maintain a chair-like transition state, intramolecular delivery of the acetate group from the enol-lead triacetate intermediate $\mathbf{1 4}$ takes place from the axial direction (Scheme 3). ${ }^{10}$ The $\mathrm{C}-6$ substituent is in an axial orientation due to $\mathrm{A}^{(1,3)}$ strain with the $N$-acyl group leading to the observed major diastereomer 15a. ${ }^{11}$ The stereochemistry of the major diastereomer 15a was confirmed by subjecting vinylogous amide $\mathbf{1 3}$ to a mixture of $\mathrm{Pb}(\mathrm{OAc})_{4}$ and $5 \% \mathrm{AcOH}$ for 3 days at reflux to give a 4:1 mixture of C-7 acetates (Scheme 4). A large NOE (9\%) between H-6 and H-7 was observed in cis-acetate 15b. No NOE was observed in the trans-acetate $15 \mathbf{a}$ between $\mathrm{H}-6$ and $\mathrm{H}-7$. In addition, $J_{\mathrm{H} 6-\mathrm{H} 7}=4.9 \mathrm{~Hz}$ in $\mathbf{1 5 b}$ and $J_{\mathrm{H} 6-\mathrm{H} 7}=2.0 \mathrm{~Hz}$ in $15 \mathbf{a}$ were observed, confirming the assignment of $15 \mathbf{a}$.

After considerable experimentation, hydrolysis of trans-acetate 15a was achieved by treatment with $\mathrm{Sc}(\mathrm{OTf})_{3}$ in a 4:1 MeOH$/ \mathrm{H}_{2} \mathrm{O}$ mixture to afford the corresponding $\alpha$-hydroxy ketone (Scheme 3). ${ }^{12,13}$ Protection of the resulting secondary alcohol as a TBS ether, followed by acetal exchange gave dihydropyridone 16 in $86 \%$ yield ( 3 steps). ${ }^{14}$ We then turned our attention to finding suitable reaction conditions to effect the conjugate allylation of compound $\mathbf{1 6}$ with the known allylsilane reagent 17. ${ }^{15}$ To our disappointment, the use of Sakurai's 
conjugate allylation conditions resulted only in the recovery of starting materials, even at temperatures as high as $-20^{\circ} \mathrm{C} .16$

In order to enhance the reactivity of the allylation reagent in the conjugate addition, we prepared the known allylstannane compound $6 .{ }^{17}$ The choice of compound $\mathbf{6}$ as the nucleophilic partner was deliberate as allylstannane reagents of this type are known to be more reactive than the corresponding allylsilanes. This is due to the presence of more anionic character on the carbon atom in the C-Sn bond, which makes it a weaker bond. ${ }^{18}$ To our delight, conjugate allylation of dihydropyridone $\mathbf{1 6}$ with stannane $\mathbf{6}$ gave the desired piperidone $\mathbf{1 9}$ as the major product in $65 \%$ yield $(d r 5.5: 1) .{ }^{19}$ The observed stereochemistry of the major diastereomer can be explained by stereoelectronic-controlled axial approach of the nucleophile, where the incoming allyl group adds syn to the axial substituent at C-6. ${ }^{20}$ The use of TMSOTf as the Lewis acid source in this type of transformation proved to be extremely important, as both the employment of $\mathrm{BF}_{3} \cdot \mathrm{OEt}_{2}$ and $\mathrm{TiCl}_{4}$ resulted in isolation of starting material 16. This, to the best of our knowledge, represents the first example of Lewis-acid mediated conjugate allylation performed on a substituted dihydropyridone to give the requisite quaternary carbon $\alpha$ to the nitrogen atom (C-1 in this case). ${ }^{21}$ The conjugate allylation is unplagued by 1,2 - addition and gives exclusively the 1,4-product. The reaction proceeds with good stereocontrol, making it an attractive way to establish quaternary centers stereoselectively in appropriately substituted dihydropyridone systems.

Next, we turned our attention to the epimerization of the C-7 silyl ether in piperidone 19, since there is a cis stereochemical relationship between the two substituents at C-6 and C-7 in the natural product (Scheme 5).

After considerable experimentation, ${ }^{22 \mathrm{a}}$ we obtained the desired cis-epimer $\mathbf{2 0}$ on treatment with DBU, but only as a 1:1 mixture of stereoisomers at C-7. In an attempt to increase the yield of the desired compound, piperidone 19 was subjected to more forcing epimerization conditions (DBU, pyridine, $\mathrm{CHCl}_{3}, 80^{\circ} \mathrm{C}$ ); however, a complex mixture of products was observed and the ratio of $\mathbf{2 0}$ to $\mathbf{1 9}$ did not improve. Apparently, our optimal conditions led to a 1:1 thermodynamic mixture of epimers at $C-7 .{ }^{23}$ At this point we decided to epimerize the $\mathrm{C}-7$ center at a later stage in the synthesis. 24

Next, hydrolysis of piperidone $\mathbf{1 9}$ in an aqueous iodomethane solution gave keto-aldehyde 21 in a quantitative yield, which was subsequently used without further purification (Scheme 6). ${ }^{25}$ We now were in position to investigate the proposed intramolecular pinacol coupling which would simultaneously set the stereocenters at C-8 and C-9 of diol 3. ${ }^{26}$ Unfortunately, after considerable effort using various conditions $\left(\mathrm{VCl}_{3} / \mathrm{Zn} ; \mathrm{TiCl}_{3} / \mathrm{Zn}-\mathrm{Cu} ; \mathrm{SmI}_{2}\right)$, we were unable to achieve the desired transformation. All attempts led to the recovery of starting materials or a complex mixture of products.

Due to our inability to obtain the desired pinacol cyclization product $\mathbf{3}$, we turned our attention to a second generation retrosynthetic approach to (-)-FR901483 (1) (Scheme 7). Our analysis suggested that the targeted compound 1 could be derived via reductive amination in ketone 22, followed by phosphorylation at C-9. The C-8/C-9 bond connectivity would this time be established by taking advantage of an intramolecular 6-exo-tet epoxide opening of vinyl bromide 23. This will again be followed by a Mitsunobu-type cyclization to construct the pyrrolidine portion of the aza-tricyclo dodecane nucleus in ketone $\mathbf{2 2}$. Vinyl bromide $\mathbf{2 3}$ in turn would arise from $\alpha$-hydroxy ketone $\mathbf{2 4}$ via a stereoselective carbonyl reduction, monomesylation of the less hindered alcohol at C-7, and epoxide formation. Piperidone $\mathbf{2 4}$ would be accessed by the conjugate addition of allyltin reagent $\mathbf{6}$ to dihydropyridone $\mathbf{2 5}$, once again providing the C-1 quaternary center stereoselectively. Vinylogous amide $\mathbf{2 5}$ would be synthesized from dihydropyridone 7 by our tandem conjugate addition/oxidative 
rearrangement protocol, followed by a regioselective installment of the vinyl bromide group and installation of the alcohol function at C-7.

Our second generation approach to the target began with exposure of dihydropyridone $\mathbf{1 1}$ to copper-mediated addition of known Grignard reagent $\mathbf{2 6}^{27}$ in the presence of trimethylsilyl chloride to give silyl enol ether 27 in a quantitative yield (Scheme 8). Subjection of 27 to premixed $o$-iodoxybenzoic acid (IBX) and 4-methoxypyridine $N$-oxide (MPO) afforded the desired dihydropyridone $\mathbf{2 8}$ in $55 \%$ yield. ${ }^{28,29}$ The versatility of this transformation is exemplified by the fact that the sensitive acetylene functionality is well tolerated under the reaction conditions. ${ }^{30}$ Removal of the terminal trimethylsilyl group in $\mathbf{2 8}$ yielded the corresponding acetylene, which was then subjected to $B$-bromo-9-BBN to yield vinyl bromide 29 as a single regioisomer. ${ }^{31}$ Reaction of $\mathbf{2 9}$ with $\mathrm{Pb}(\mathrm{OAc})_{4}$ resulted in the corresponding trans-acetate. ${ }^{32} \mathrm{Sc}(\mathrm{OTf})_{3}$-mediated hydrolysis of the acetate group was followed by protection of the resulting secondary alcohol as the TBS ether to yield dihydropyridone $\mathbf{2 5}$ (57\%, 3 steps). Conjugate allylation with allylstannane $\mathbf{6}$ gave the desired trimethylsilyl enol ether $\mathbf{3 0}$ as the a single diastereomer at C-1. Interesting, compound $\mathbf{3 0}$ proved to be stable to silica gel chromatography and had to be subjected to acidic hydrolysis to yield the desired piperidone 31 in $85 \%$ yield ( 2 steps). We proceeded to establish the stereochemical relationship between the allylic group at $\mathrm{C}-1$ and the benzylic substituent at C-6 in compound 31. Irradiation of $\mathrm{H}_{6}$ showed a large $\mathrm{NOE}$ correlation to $\mathrm{H}_{11}(5.8 \%)$, thus confirming the assigned stereochemistry. This result confirmed our working model that the conjugate addition of allylstannane 6 to C-6 substituted dihydropyridones indeed operated under stereoelectronicapproach control, where the incoming allylgroup adds syn to the axial substituent at C-6 in order to maintain a chair-like transition state. 20

Having successfully installed the C-1 quaternary center with the desired stereochemical orientation, we then focused on the synthesis of key epoxide intermediate $\mathbf{2 3}$ (Scheme 9). Deprotection of the TBS ether in $\mathbf{3 1}$ was followed by intramolecular, stereocontrolled reduction at C-8 with tetramethylammonium triacetoxyborohydride to give the 1,2-diol $\mathbf{3 2}$ as a single stereoisomer. The selective derivatization of the $\mathrm{C}-8$ position of trans-diol 32 by reaction with benzoic anhydride yielded the $\mathrm{C}-8$ benzoate ester $\mathbf{3 3}$ as the major product of a 3:1 mixture of C-8/C-7 regioisomers. ${ }^{33}$ The direction of the acylation was determined by examination of the ${ }^{1} \mathrm{H}$ NMR spectra of diol 32 and benzoate ester 33. The $\mathrm{H}_{8}$ hydrogen atom frequency in 33 shifted downfield as compared to the chemical shift of $\mathrm{H}_{7}$. This is to be expected as the electron withdrawing benzoate ester group exerts a larger deshielding effect on the $\mathrm{H}_{8}$ than on the $\mathrm{H}_{7}$ causing the observed downfield shift in its resonance. During the course of the acylation we also discovered that subjection of the corresponding undesired C-7 benzoate ester to methanolic $\mathrm{NaOH}$ resulted in the quantitative formation of diol 32, allowing the $\mathrm{C}-7$ isomer to be recycled. Mesylation of $\mathbf{3 3}$ was followed by subjection of the benzoate ester moiety in 34 to basic hydrolysis to give the tetrahedral intermediate 35, which upon collapse yielded the desired epoxide $\mathbf{2 3}$ in a one-pot event. 34

With epoxide 23 in hand, we turned our attention to finding suitable conditions to afford the C-8/C- 9 bond connectivity in FR901483 by effecting an intramolecular epoxide ring opening (Scheme 10). This type of intramolecular opening of an epoxide with an aryllithium to form a six-membered ring has been reported. ${ }^{35}$ Unfortunately, all our efforts to achieve the desired 6-exo-tet-epoxide opening to afford product 36 were unsuccessful. ${ }^{22 \mathrm{~b}}$ Exposure to $n$ butyllithium or dibutylcopper lithium led to the recovery of starting materials together with products resulting from attack at the carbamate. Similar results were observed when tertbutyllithium was used at $-78^{\circ} \mathrm{C}$. Gratifyingly, lithium bromide exchange was achieved by reaction with $t$-BuLi at $-120{ }^{\circ} \mathrm{C}$ for 1 hour; however, all attempts to obtain the desired epoxide opening product 36 via formation of a higher-order cuprate or a lower-order cuprate gave the corresponding debrominated product exclusively. A likely reason for the cyclization failure is 
an inherent $\mathrm{A}^{(1,3)}$ strain in the molecule that produces a conformation unfavorable to the desired bond formation.

\section{Summary}

Although the investigated routes to the bicyclic [3.3.1] ring system of FR901483 did not result in the formation of the C-8/C-9 bond connectivity, diastereoselective methodology for the installation of the quaternary center at $\mathrm{C}-1$ utilizing a conjugate allylation reaction was developed, and several highly substituted piperidine derivatives were prepared regio- and stereoselectively. Most of the stereoselective transformations were designed based on a conformational bias caused by $\mathrm{A}^{(1,3)}$ strain present in the molecules. Unfortunately, failure of the attempted C-8/C-9 formation is also attributed to allylic strain. If an $\mathrm{N}$-acyl group of a late intermediate can be selectively removed to relieve $\mathrm{A}^{(1,3)}$ strain, ring-closure may be feasible. Further studies will be needed to determine if the synthetic routes attempted can be modified to allow the key bond formation.

\section{Experimental Section}

\section{6-(2-[1,3]Dioxolan-2-yl-ethyl)-2-(4-methoxybenzyl)-4-oxo-3,4-dihydro-2H-pyridine-1- carboxylic acid phenyl ester (13)}

Magnesium turnings $(7.57 \mathrm{~g}, 311 \mathrm{mmol})$ were mechanically activated by stirring at rt overnight under argon, and then $50 \mathrm{~mL}$ of anhydrous THF was added. A portion of 2-(2-bromoethyl)-1,3dioxolane $(9.7 \mathrm{~mL}, 83 \mathrm{mmol})$ in $50 \mathrm{~mL}$ of anhydrous THF was slowly added to the mixture. Once the reaction was initiated, the mixture was cooled to $10{ }^{\circ} \mathrm{C}$, and the remaining bromide was continuously added over a period of $2 \mathrm{~h}$. After the addition was complete, the reaction mixture was stirred at $10{ }^{\circ} \mathrm{C}$ for $9 \mathrm{~h}$ to form the corresponding Grignard reagent. In a separate flask, copper bromide dimethyl sulfide complex $(22.6 \mathrm{~g}, 110 \mathrm{mmol})$ was mixed with $50 \mathrm{~mL}$ of freshly distilled THF under argon and cooled to $-78^{\circ} \mathrm{C}$. A solution of 2-(4-

methoxybenzyl)-4-oxo-3,4-dihydro-2H-pyridine-1-carboxylic acid phenyl ester (11) (7.40 g, $20.7 \mathrm{mmol})$ in THF $(30 \mathrm{~mL})$ was added, followed by a premixed solution of TMSCl $(30.6 \mathrm{~mL}$, $241 \mathrm{mmol})$ and $\mathrm{Et}_{3} \mathrm{~N}(35.2 \mathrm{~mL}, 252 \mathrm{mmol})$. A solution of the above prepared Grignard reagent in THF was then added dropwise over a period of $1 \mathrm{~h}$. Once the addition was complete, the resulting slurry was stirred at $-78^{\circ} \mathrm{C}$ for $2 \mathrm{~h}$ and then warmed to $-45^{\circ} \mathrm{C}$. After $24 \mathrm{~h}$, the reaction mixture was quenched with a carefully buffered solution of $100 \mathrm{~mL}$ of $\mathrm{NH}_{4} \mathrm{OH}$ and $\mathrm{NH}_{4} \mathrm{Cl}$ $(\mathrm{pH} 8)$, and the phases were separated. The aqueous phase was extracted with $\mathrm{Et}_{2} \mathrm{O}(3 \times 150$ $\mathrm{mL}$ ), and the combined organic layers were washed with $10 \% \mathrm{NH}_{4} \mathrm{OH}$ until no further blue color was observed in the aqueous layer. The organic layers were combined, dried over $\mathrm{K}_{2} \mathrm{CO}_{3}$, filtered through Celite, and concentrated in vacuo to yield crude silyl enol ether $\mathbf{1 2}$.

To a solution of the above enol ether 12 in $100 \mathrm{~mL}$ of anhydrous $\mathrm{CH}_{3} \mathrm{CN}$ under argon was added $\mathrm{Pd}(\mathrm{OAc})_{2}(15 \mathrm{~mol} \%, 0.74 \mathrm{~g}, 3.3 \mathrm{mmol})$, followed by $\mathrm{CuCl}(2.95 \mathrm{~g}, 29.8 \mathrm{mmol})$. The solution was purged with oxygen, warmed to $60^{\circ} \mathrm{C}$ under an $\mathrm{O}_{2}$-balloon pressure atmosphere, and stirred vigorously overnight. Upon completion, the reaction mixture was cooled to rt, filtered through Celite with $\mathrm{CH}_{2} \mathrm{Cl}_{2}$, and the solvent was removed in vacuo to yield the crude product 13. Purification by silica gel chromatography (20 to $30 \%$ EtOAc in hexanes) gave $6.75 \mathrm{~g}(70 \%, 2$ steps) of the desired product 13 as a yellow foam: IR (neat) 2922, 2851, 1719, $1512,1494,1453,1248,1203,834 \mathrm{~cm}^{-1 ;}{ }_{1} \mathrm{H}$ NMR $\left(\mathrm{CDCl}_{3}, 300 \mathrm{MHz}\right) \delta 7.38(\mathrm{dt}, J=1.2 \mathrm{~Hz}$, $5.7 \mathrm{~Hz}, 2 \mathrm{H}), 7.26(\mathrm{~d}, J=3.9 \mathrm{~Hz}, 1 \mathrm{H}), 7.10(\mathrm{~d}, J=6.0 \mathrm{~Hz}, 2 \mathrm{H}) 7.00(\mathrm{~d}, J=6.0 \mathrm{~Hz}, 2 \mathrm{H}), 6.85$ $(\mathrm{d}, J=6.9 \mathrm{~Hz}, 2 \mathrm{H}), 5.63(\mathrm{~s}, 1 \mathrm{H}), 5.07(\mathrm{q}, J=5.1 \mathrm{~Hz}, 1 \mathrm{H}), 4.93(\mathrm{t}, J=3.3 \mathrm{~Hz}, 1 \mathrm{H}), 3.96(\mathrm{~m}$, $2 \mathrm{H}), 3.86(\mathrm{~m}, 2 \mathrm{H}), 3.79(\mathrm{~s}, 3 \mathrm{H}), 3.08(\mathrm{~m}, 2 \mathrm{H}), 2.81(\mathrm{~m}, 3 \mathrm{H}), 2.43(\mathrm{~d}, J=12.6 \mathrm{~Hz}, 1 \mathrm{H}), 1.97$ $(\mathrm{m}, 2 \mathrm{H}) ;{ }^{13} \mathrm{C} \mathrm{NMR}\left(\mathrm{CDCl}_{3}, 75 \mathrm{MHz}\right) \delta 193.0,158.5,(157.3 \mathrm{r}), 151.5,150.3,130.5,129.4$, 128.8, 126.1, 121.3, 114.0, 113.2, 103.2, 64.9, 57.0, 57.2, 55.2, 39.7, 35.5, 32.3, 30.1; HRMS $(\mathrm{M}+\mathrm{H})^{+}$calcd for $\mathrm{C}_{25} \mathrm{H}_{27} \mathrm{NO}_{6}+\mathrm{H}: 438.1917$, found 438.1904 . 
$\left(2 S^{\star}, 3 S^{\star}\right)-3-A c e t o x y-6-(2-[1,3]$ dioxolan-2-yl-ethyl)-2-(4-methoxybenzyl)-4-oxo-3,4-dihydro$2 \mathrm{H}$-pyridine-1-carboxylic acid phenyl ester (15a)

To a stirred solution of $\mathbf{1 3}$ (121 $\mathrm{mg}, 0.277 \mathrm{mmol}$ ) in $8 \mathrm{~mL}$ of toluene at $\mathrm{rt}$ was added lead (IV) acetate $(160 \mathrm{mg}, 0.36 \mathrm{mmol})$. The resulting mixture was refluxed for $3 \mathrm{~h}$, cooled to rt, and additional lead (IV) acetate $(160 \mathrm{mg}, 0.36 \mathrm{mmol})$ was added. The mixture was refluxed for 3 h. After cooling to rt, the solution was filtered through Celite with $\mathrm{CH}_{2} \mathrm{Cl}_{2}$. The solution was washed with saturated aqueous $\mathrm{NaHCO}_{3}(30 \mathrm{~mL})$, and the aqueous phase extracted with $\mathrm{CH}_{2} \mathrm{Cl}_{2}(3 \times 30 \mathrm{~mL})$. The combined organic phase was dried over $\mathrm{Na}_{2} \mathrm{SO}_{4}$, filtered through Celite, and concentrated in vacuo to yield crude 15a. Purification by silica gel chromatography (20 to $30 \%$ EtOAc in hexanes) gave $103 \mathrm{mg}$ (75\%) of 15a as a colorless oil: IR (neat) 2934, $1741,1678,1592,1513,1401,1248,1181,1032,911,750 \mathrm{~cm}^{-1 ;}{ }^{\mathrm{H}} \mathrm{NMR}\left(\mathrm{CDCl}_{3}, 300 \mathrm{MHz}\right)$ $\delta 7.36(\mathrm{t}, J=7.4 \mathrm{~Hz}, 2 \mathrm{H}), 7.22(\mathrm{~d}, J=7.3 \mathrm{~Hz}, 1 \mathrm{H}), 7.12(\mathrm{~d}, J=8.3 \mathrm{~Hz}, 2 \mathrm{H}), 6.86(\mathrm{t}, J=8.7$ $\mathrm{Hz}, 4 \mathrm{H}), 5.70(\mathrm{~s}, 1 \mathrm{H}), 5.23(\mathrm{dt}, J=1.5,6.6 \mathrm{~Hz}, 1 \mathrm{H}), 4.93(\mathrm{~m}, 2 \mathrm{H}), 3.96(\mathrm{~m}, 2 \mathrm{H}), 3.86(\mathrm{~m}, 2$ H), $3.79(\mathrm{~s}, 3 \mathrm{H}), 3.13(\mathrm{~m}, 1 \mathrm{H}), 2.97(\mathrm{~m}, 2 \mathrm{H}), 2.82(\mathrm{~m}, 1 \mathrm{H}), 2.09(\mathrm{~s}, 3 \mathrm{H}), 2.01(\mathrm{~m}, 2$ $\mathrm{H}) ;{ }^{13} \mathrm{C}$ NMR $\left(\mathrm{CDCl}_{3}, 75 \mathrm{MHz}\right) \delta 187.6,169.9,159.4,(159.0 \mathrm{r}), 151.9,150.3,130.6,129.7$, 127.8, 126.5, 121.3, 114.4, 112.1, 103.4, 71.4, 65.2, 65.1, 61.9, 55.4, 33.5, 32.3, 30.5, 21.0; HRMS $(\mathrm{M}+\mathrm{H})^{+}$calcd for $\mathrm{C}_{27} \mathrm{H}_{29} \mathrm{NO}_{8}+\mathrm{H}: 496.1971$, found 496.1959 .

$\left(2 S^{\star}, 3 R^{\star}\right)-3-A c e t o x y-6-(2-[1,3] d i o x o l a n-2-y l-e t h y l)-2-(4-m e t h o x y b e n z y l)-4-o x o-3,4-d i h y d r o-$ $2 H$-pyridine-1-carboxylic acid phenyl ester (15b)

IR (neat) 2934, 1738, 1682, 1593, 1513, 1397, 1289, 1249, 1177, 1033, $913 \mathrm{~cm}^{-1 ;}{ }_{1} \mathrm{H} \mathrm{NMR}$ $\left(\mathrm{CDCl}_{3}, 300 \mathrm{MHz}\right) \delta 7.24(\mathrm{~m}, 5 \mathrm{H}), 6.86(\mathrm{~d}, J=6.3 \mathrm{~Hz}, 2 \mathrm{H}), 6.72(\mathrm{~d}, J=6.3 \mathrm{~Hz}, 2 \mathrm{H}), 5.83$ $(\mathrm{d}, J=4.5 \mathrm{~Hz}, 1 \mathrm{H}), 5.63(\mathrm{~s}, 1 \mathrm{H}), 5.25(\mathrm{~m}, 1 \mathrm{H}), 4.91(\mathrm{t}, J=3.3 \mathrm{~Hz}, 1 \mathrm{H}), 3.96(\mathrm{~m}, 2 \mathrm{H}), 3.84$ $(\mathrm{m}, 2 \mathrm{H}), 3.79(\mathrm{~m}, 3 \mathrm{H}), 2.98(\mathrm{~m}, 3 \mathrm{H}), 2.81(\mathrm{~m}, 1 \mathrm{H}), 2.19(\mathrm{~s}, 3 \mathrm{H}), 2.05(\mathrm{~m}, 1 \mathrm{H}), 1.90(\mathrm{~m}, 1$ $\mathrm{H}) ;{ }^{13} \mathrm{C} \mathrm{NMR}\left(\mathrm{CDCl}_{3}, 75 \mathrm{MHz}\right) \delta 189.1,169.9,158.7$ (158.8 r), 151.3, 150.3, 130.7, 129.6, 129.1, 126.4, 121.5, 114.2, 111.6, 103.4, 73.0, 65.2, 65.1, 60.7, 55.5, 32.4, 30.7, 30.0, 20.9; HRMS $(\mathrm{M}+\mathrm{H})^{+}$calcd for $\mathrm{C}_{27} \mathrm{H}_{29} \mathrm{NO}_{8}+\mathrm{H}: 496.1971$, found 496.1961 .

\section{$\left(2 S^{\star}, 3 S^{\star}\right)-3-($ tert-Butyldimethylsilanyloxy)-6-(2-[1,3]dithian-2-yl-ethyl)-2-(4-methoxybenzyl)- 4-oxo-3,4-dihydro-2H-pyridine-1-carboxylic acid phenyl ester (16)}

To a solution of $15 \mathbf{a}(23.0 \mathrm{mg}, 0.046 \mathrm{mmol})$ in $\mathrm{H}_{2} \mathrm{O}: \mathrm{MeOH}(1.0 \mathrm{~mL}, 1: 4)$ was added $\mathrm{ScOTf}_{3}$ $(20 \mathrm{~mol} \%, 5.0 \mathrm{mg}, 0.0093 \mathrm{mmol})$. The resulting mixture was stirred at $\mathrm{rt}$ for $40 \mathrm{~h}$. Upon completion, the reaction mixture was poured into $\mathrm{H}_{2} \mathrm{O}(5 \mathrm{~mL})$, and extracted with $\mathrm{CH}_{2} \mathrm{Cl}_{2}$ (3 $\times 10 \mathrm{~mL}$ ). The combined extracts were dried over $\mathrm{Na}_{2} \mathrm{SO}_{4}$, filtered through Celite with $\mathrm{CH}_{2} \mathrm{Cl}_{2}$, and the solvent removed in vacuo. Purification by silica gel chromatography (20 to $30 \%$ EtOAc in hexanes) gave $21.0 \mathrm{mg}(100 \%)$ of the desired $\alpha$-hydroxy ketone as a white solid which was used directly in the next step. Mp 152-154 ${ }^{\circ} \mathrm{C}$; IR (neat) 3394, 2919, 1737 , 1664, 1513, 1410, 1320, 1248, 1179, $1033 \mathrm{~cm}^{-1 ; 1} \mathrm{H} \mathrm{NMR}\left(\mathrm{CDCl}_{3}, 300 \mathrm{MHz}\right) \delta 7.40(\mathrm{t}, J=$ $5.7 \mathrm{~Hz}, 1 \mathrm{H}), 7.27(\mathrm{~d}, J=3.9 \mathrm{~Hz}, 2 \mathrm{H}), 7.10(\mathrm{~d}, J=6.3 \mathrm{~Hz}, 2 \mathrm{H}), 7.01(\mathrm{~d}, J=6.0 \mathrm{~Hz}, 2 \mathrm{H}), 6.83$ $(\mathrm{d}, J=6.9 \mathrm{~Hz}, 2 \mathrm{H}), 5.60(\mathrm{~s}, 1 \mathrm{H}), 5.09(\mathrm{dt}, J=2.1,8.1 \mathrm{~Hz}, 1 \mathrm{H}), 4.92(\mathrm{t}, J=7.2 \mathrm{~Hz}, 1 \mathrm{H}), 3.96$ (m, $2 \mathrm{H}), 3.87(\mathrm{~m}, 2 \mathrm{H}), 3.79(\mathrm{~s}, 3 \mathrm{H}), 3.04(\mathrm{~m}, 2 \mathrm{H}), 2.81(\mathrm{~m}, 2 \mathrm{H}), 2.70(\mathrm{~d}, J=4.2 \mathrm{~Hz}, 1 \mathrm{H})$, 1.97 (m, $2 \mathrm{H}) ;{ }^{13} \mathrm{C} \mathrm{NMR}\left(\mathrm{CDCl}_{3}, 75 \mathrm{MHz}\right) \delta 192.9,158.4(159.2 \mathrm{r}), 152.2,150.4,130.6,129.6$, $128.4,126.3,121.5,114.3,110.3,103.4,70.7,65.1,65.0,64.2,55.4,33.5,32.3,30.5 ;$ HRMS $(\mathrm{M}+\mathrm{H})^{+}$calcd for $\mathrm{C}_{25} \mathrm{H}_{27} \mathrm{NO}_{7}+\mathrm{H}: 454.1866$, found 454.1873 .

To a solution of the above $\alpha$-hydroxy ketone $(550 \mathrm{mg}, 1.22 \mathrm{mmol})$ in DMF $(20 \mathrm{~mL})$ at $20^{\circ} \mathrm{C}$ was added imidazole (165 mg, $2.43 \mathrm{mmol})$ and tert-butyldimethylsilyl chloride ( $275 \mathrm{mg}, 1.82$ $\mathrm{mmol})$. The resulting mixture was stirred at $\mathrm{rt}$ for $12 \mathrm{~h}$. The reaction mixture was quenched with saturated aqueous $\mathrm{NaHCO}_{3}(50 \mathrm{~mL})$ and extracted with $\mathrm{CH}_{2} \mathrm{Cl}_{2}(3 \times 40 \mathrm{~mL})$. The combined organic layers were washed with brine $(30 \mathrm{~mL})$, dried over $\mathrm{Na}_{2} \mathrm{SO}_{4}$, filtered through Celite with $\mathrm{CH}_{2} \mathrm{Cl}_{2}$, and the solvent was removed in vacuo. Purification by silica gel chromatography (30 to $40 \%$ EtOAc in hexanes) gave $620 \mathrm{mg}$ (90\%) of the desired TBS- 
protected alcohol as a colorless oil which was used directly in the next step. IR (neat) 2953,

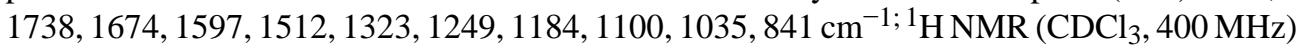
$\delta 7.37(\mathrm{dt}, J=1.0,7.4 \mathrm{~Hz}, 2 \mathrm{H}), 7.23(\mathrm{t}, J=8.4 \mathrm{~Hz}, 1 \mathrm{H}), 7.10(\mathrm{~d}, J=8.4 \mathrm{~Hz}, 2 \mathrm{H}), 6.95$ (d, $J=7.7 \mathrm{~Hz}, 2 \mathrm{H}), 6.85(\mathrm{~d}, J=6.6 \mathrm{~Hz}, 2 \mathrm{H}), 5.59$ (s, $1 \mathrm{H}), 5.09$ (dt, $J=2.0,7.2 \mathrm{~Hz}, 1 \mathrm{H}), 4.92$ (t, $J=4.4 \mathrm{~Hz}, 1 \mathrm{H}), 3.95(\mathrm{~m}, 2 \mathrm{H}), 3.84(\mathrm{~m}, 2 \mathrm{H}), 3.79(\mathrm{~s}, 3 \mathrm{H}), 3.69(\mathrm{~s}, 1 \mathrm{H}), 3.11(\mathrm{~m}, 1 \mathrm{H})$, $3.01(\mathrm{~m}, 1 \mathrm{H}), 2.85(\mathrm{~m}, 2 \mathrm{H}), 1.99(\mathrm{~m}, 2 \mathrm{H}), 0.83(\mathrm{~s}, 9 \mathrm{H}), 0.07$ (s, $3 \mathrm{H}), 0.02(\mathrm{~s}, 3 \mathrm{H}) ;{ }^{13} \mathrm{C} \mathrm{NMR}$ $\left(\mathrm{CDCl}_{3}, 100 \mathrm{MHz}\right) \delta 191.9,157.8$ (158.8 r), 152.5, 150.5, 130.5, 129.7, 128.7, 126.3, 121.6, 114.3, 111.0, 103.6, 71.6, 65.7, 65.2, 65.1, 55.5, 33.2, 32.5, 30.5, 25.8, 18.2, -4.6, -5.0; HRMS $(\mathrm{M}+\mathrm{H})^{+}$calcd for $\mathrm{C}_{31} \mathrm{H}_{41} \mathrm{NO}_{7} \mathrm{Si}+\mathrm{H}: 568.2731$, found 568.2741.

To a solution of the above TBS-protected alcohol (620 mg, $1.04 \mathrm{mmol})$ in $\mathrm{CH}_{2} \mathrm{Cl}_{2}(10 \mathrm{~mL})$ at $20{ }^{\circ} \mathrm{C}$ was added neat $\mathrm{BF}_{3} \cdot \mathrm{OEt}_{2}(60 \mu \mathrm{l}, 0.44 \mathrm{mmol})$ and then 1,3-propanedithiol $(170 \mu \mathrm{l}, 1.64$ $\mathrm{mmol})$. The resulting mixture was stirred at $\mathrm{rt}$ for $8 \mathrm{~h}$. The reaction mixture was quenched with saturated aqueous $\mathrm{NaHCO}_{3}(50 \mathrm{~mL})$ and extracted with $\mathrm{CH}_{2} \mathrm{Cl}_{2}(3 \times 40 \mathrm{~mL})$. The combined organic layers were washed with brine, dried over $\mathrm{Na}_{2} \mathrm{SO}_{4}$, filtered through Celite with $\mathrm{CH}_{2} \mathrm{Cl}_{2}$, and the solvent was removed in vacuo to yield the crude product 16. Purification by silica gel chromatography (10 to 20\% EtOAc in hexanes) gave $637 \mathrm{mg}$ (95\%) of 16 as a colorless oil: IR (neat) 2929, 1738, 1673, 1597, 1249, 1182, 1096, $837 \mathrm{~cm}^{-1 ; 1} \mathrm{H}$ NMR $\left(\mathrm{CDCl}_{3}, 300 \mathrm{MHz}\right) \delta 7.37(\mathrm{t}, J=7.6 \mathrm{~Hz}, 2 \mathrm{H}), 7.22(\mathrm{t}, J=6.8 \mathrm{~Hz}, 1 \mathrm{H}), 7.10(\mathrm{~d}, J=8.4 \mathrm{~Hz}, 2$ H), $6.92(\mathrm{~d}, J=6.4 \mathrm{~Hz}, 2 \mathrm{H}), 6.84(\mathrm{~d}, J=6.8 \mathrm{~Hz}, 2 \mathrm{H}), 5.56(\mathrm{~s}, 1 \mathrm{H}), 4.99$ (dt, J= 3.2, $11.2 \mathrm{~Hz}$, $1 \mathrm{H}), 4.00(\mathrm{t}, J=9.6 \mathrm{~Hz}, 1 \mathrm{H}), 3.80(\mathrm{~s}, 3 \mathrm{H}), 3.71(\mathrm{~d}, J=2.4 \mathrm{~Hz}, 1 \mathrm{H}), 3.19(\mathrm{~m}, 1 \mathrm{H}), 2.91(\mathrm{~m}$, $1 \mathrm{H}), 2.81$ (m, $6 \mathrm{H}), 2.05(\mathrm{~m}, 3 \mathrm{H}), 1.97$ (m, $1 \mathrm{H}), 0.84$ (s, $9 \mathrm{H}), 0.04$ (s, $3 \mathrm{H}), 0.00$ (s, 3 $\mathrm{H}) ;{ }^{13} \mathrm{C} \mathrm{NMR}\left(\mathrm{CDCl}_{3}, 100 \mathrm{MHz}\right) \delta 191.8,158.9,157.0,152.4,150.6,130.5,129.7,128.54$, 126.3, 121.6, 114.4, 111.2, 71.7, 65.5, 55.5, 46.7, 34.1, 33.2, 33.1, 30.3, 26.0, 25.8, 18.3, -4.6, -5.0 ; HRMS $(\mathrm{M}+\mathrm{H})^{+}$calcd for $\mathrm{C}_{32} \mathrm{H}_{43} \mathrm{NO}_{5} \mathrm{~S}_{2} \mathrm{Si}+\mathrm{H}$ : 614.2430, found 614.2439.

\section{$\left(2 R^{\star}, 5 S^{\star}, 6 S^{\star}\right)$-2(2-Benzyloxymethylallyl)-5-(tert-butyldimethylsilanyloxy)-2-(2-[1,3]dithian-2- yl-ethyl)-6-(4-methoxybenzyl)-4-oxo-piperidine-1-carboxylic acid phenyl ester (19)}

A well-stirred solution of dihydropyridone $16(0.560 \mathrm{~g}, 0.912 \mathrm{mmol})$ in $20 \mathrm{~mL}$ of dry $\mathrm{CH}_{2} \mathrm{Cl}_{2}$ was cooled to $-78{ }^{\circ} \mathrm{C}$. TMSOTf $(490 \mu \mathrm{l}, 2.73 \mathrm{mmol})$ was added in one portion, giving rise to a deep orange color. After $5 \mathrm{~min}$, neat (2-benzyloxymethylallyl)tributylstannane reagent 6 (1.00 g, $1.82 \mathrm{mmol}$ ) was added dropwise over a $10 \mathrm{~min}$ period. The reaction mixture was stirred for $4 \mathrm{~h}$ at $-78{ }^{\circ} \mathrm{C}$, warmed up to $-45^{\circ} \mathrm{C}$, and stirred for additional $6 \mathrm{~h}$. The reaction mixture was quenched with saturated aqueous $\mathrm{NaHCO}_{3}(50 \mathrm{~mL})$ and warmed to rt, during which time it became colorless. The mixture was extracted with $\mathrm{CH}_{2} \mathrm{Cl}_{2}(3 \times 100 \mathrm{~mL})$. The organic extracts were dried over $\mathrm{Na}_{2} \mathrm{SO}_{4}$ and filtered through Celite. The solvent was removed in vacuo to yield the crude product 19. Purification by silica chromatography ( 0 to $20 \%$ EtOAc in hexanes) gave $458 \mathrm{mg}(65 \%)$ of $\mathbf{1 9}$ as a colorless oil: IR (neat) 2929, 2856, 1720, 1513, 1348, 1250, 1201, 1098, $838 \mathrm{~cm}^{-1 ;}{ }^{1} \mathrm{H} \mathrm{NMR}\left(\mathrm{CDCl}_{3}, 300 \mathrm{MHz}\right) \delta 7.39(\mathrm{~m}, 12 \mathrm{H}), 6.82(\mathrm{~d}, J$ $=9.2 \mathrm{~Hz}, 2 \mathrm{H}), 5.34(\mathrm{~d}, J=1.2 \mathrm{~Hz}, 1 \mathrm{H}), 5.08$ (s, $1 \mathrm{H}), 4.67$ (dd, $J=1.8,10.8 \mathrm{~Hz}, 1 \mathrm{H}), 4.50$ (s, $2 \mathrm{H}), 4.00(\mathrm{t}, J=6.4 \mathrm{~Hz}, 1 \mathrm{H}), 3.96(\mathrm{~s}, 2 \mathrm{H}), 3.78(\mathrm{~s}, 3 \mathrm{H}), 3.74(\mathrm{~s}, 1 \mathrm{H}), 3.21$ (bs, $1 \mathrm{H}), 3.19$ (d, $J=14.0 \mathrm{~Hz}, 1 \mathrm{H}), 2.98$ (bs, $1 \mathrm{H}), 2.80(\mathrm{~m}, 6 \mathrm{H}), 2.52$ (bs, $1 \mathrm{H}), 2.13$ (m, $2 \mathrm{H}), 1.90$ (m, 2 $\mathrm{H}), 1.85(\mathrm{~m}, 2 \mathrm{H}), 0.78(\mathrm{~s}, 9 \mathrm{H}),-0.10(\mathrm{~s}, 3 \mathrm{H}),-0.24(\mathrm{~s}, 3 \mathrm{H}) ;{ }^{13} \mathrm{C} \mathrm{NMR}\left(\mathrm{CDCl}_{3}, 75 \mathrm{MHz}\right) \delta$ 206.3 158.8, 151.1, 141.8, 141.4, 138.5, 130.3, 129.7, 129.4, 128.6, 128.5, 127.6, 125.9, 122.2, $120.7,114.4,73.9,72.5,71.3,70.6,64.2,55.5,47.4,30.8,30.4,30.3,30.2,26.1,25.8,25.6$, 18.1, -4.9, -5.6; HRMS $(\mathrm{M}+\mathrm{H})^{+}$calcd for $\mathrm{C}_{43} \mathrm{H}_{57} \mathrm{NO}_{6} \mathrm{~S}_{2} \mathrm{Si}+\mathrm{H}: 776.3475$, found 776.3439 .

$\left(2 R^{\star}, 5 R^{\star}, 6 S^{\star}\right)-2-(2-B e n z y l o x y m e t h y l a l l y l)-5-(t e r t-b u t y l d i m e t h y l s i l a n y l o x y)-2-(2-[1,3] d i t h i a n-$ 2-yl-ethyl)-6-(4-methoxybenzyl)-4-oxo-piperidine-1-carboxylic acid phenyl ester (20)

Compound 19 (120 mg, $0.155 \mathrm{mmol})$ in $\mathrm{CH}_{2} \mathrm{Cl}_{2}(5 \mathrm{~mL})$ was treated with pyridine $(1 \mu \mathrm{l}, 0.02$ $\mathrm{mmol})$ and $\mathrm{DBU}(20 \mu \mathrm{l}, 0.077 \mathrm{mmol})$, and the resulting mixture was refluxed for $60 \mathrm{~h}$. The solution was cooled to $\mathrm{rt}$ and quenched with $1 \mathrm{~N}$ aqueous $\mathrm{HCl}(1 \mathrm{~mL})$. The mixture was 
extracted with $\mathrm{CH}_{2} \mathrm{Cl}_{2}(3 \times 15 \mathrm{~mL})$, and combined organic extracts were dried over $\mathrm{Na}_{2} \mathrm{SO}_{4}$, filtered, and concentrated in vacuo. The crude product, a 1:1 mixture of piperidones $\mathbf{1 9}$ and 20, was purified by silica gel chromatography ( 10 in $20 \%$ EtOAc in hexanes) to give $60 \mathrm{mg}$ (50\%) of the desired product $\mathbf{2 0}$ as a colorless oil: IR (neat) 2927, 2853, 1717, 1512, 1248, 1202, 1161, 1110, $838 \mathrm{~cm}^{-1 ;}{ }_{1} \mathrm{H} \mathrm{NMR}\left(\mathrm{CDCl}_{3}, 300 \mathrm{MHz}\right) \delta 7.32(\mathrm{~s}, 8 \mathrm{H}), 7.20(\mathrm{~d}, J=9.2 \mathrm{~Hz}$, $2 \mathrm{H}), 6.80(\mathrm{~d}, J=9.2 \mathrm{~Hz}, 2 \mathrm{H}), 6.62(\mathrm{bs}, 1 \mathrm{H}), 5.34(\mathrm{~d}, J=1.6 \mathrm{~Hz}, 1 \mathrm{H}), 5.02(\mathrm{~s}, 1 \mathrm{H}), 4.93(\mathrm{~m}$, $1 \mathrm{H}), 4.62(\mathrm{~d}, J=5.6 \mathrm{~Hz}, 1 \mathrm{H}), 4.50(\mathrm{~s}, 2 \mathrm{H}), 3.99(\mathrm{t}, J=7.6 \mathrm{~Hz}, 1 \mathrm{H}), 3.92(\mathrm{~s}, 2 \mathrm{H}), 3.74(\mathrm{~s}, 3$ H), $3.06(\mathrm{bs}, 1 \mathrm{H}), 3.04(\mathrm{~d}, J=14.4 \mathrm{~Hz}, 1 \mathrm{H}), 2.86(\mathrm{~m}, 6 \mathrm{H}), 2.67(\mathrm{~d}, J=19.2 \mathrm{~Hz}, 1 \mathrm{H}), 2.59$ $(\mathrm{dt}, J=3.6,12.4 \mathrm{~Hz}, 1 \mathrm{H}), 2.48(\mathrm{~m}, 1 \mathrm{H}), 2.13(\mathrm{~m}, 1 \mathrm{H}), 1.87(\mathrm{~m}, 5 \mathrm{H}), 0.84(\mathrm{~s}, 9 \mathrm{H}), 0.08(\mathrm{~s}$, $3 \mathrm{H}), 0.00$ (s, $3 \mathrm{H}) ;{ }^{13} \mathrm{C} \mathrm{NMR}\left(\mathrm{CDCl}_{3}, 100 \mathrm{MHz}\right) \delta 206.1,158.4,150.8,142.2,138.1,131.2$, 130.7, 129.7, 129.4, 128.6, 127.9, 125.7, 122.2, 122.0, 118.2, 114.2, 75.8, 73.5, 72.8, 62.2, 60.4, 55.6, 47.5, 47.0, 37.8, 31.2, 30.6, 26.2, 25.9, 18.6, 0.2, -4.4, -5.4; HRMS $(\mathrm{M}+\mathrm{H})^{+}$calcd for $\mathrm{C}_{43} \mathrm{H}_{57} \mathrm{NO}_{6} \mathrm{~S}_{2} \mathrm{Si}+\mathrm{H}: 776.3475$, found 776.3439 .

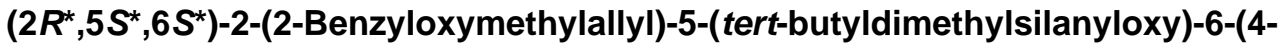 methoxybenzyl)-4-oxo-2-(3-oxo-propyl)-piperidine-1-carboxylic acid phenyl ester (21)}

To a mixture of $19(32 \mathrm{mg}, 0.41 \mathrm{mmol})$ and calcium carbonate $(100 \mathrm{mg}, 0.743 \mathrm{mmol})$ in aqueous $\mathrm{H}_{2} \mathrm{O}: \mathrm{CH}_{3} \mathrm{CN}(2.0 \mathrm{~mL}, 1: 9)$ was added $\mathrm{CH}_{3} \mathrm{I}(2.0 \mathrm{~mL}, 33 \mathrm{mmol})$. The mixture was stirred at room temperature overnight, diluted with EtOAc $(10 \mathrm{~mL})$, and filtered through Celite. The filtrate was washed with brine $(2 \times 10 \mathrm{~mL})$, dried over $\mathrm{Na}_{2} \mathrm{SO}_{4}$, and the solvent was removed in vacuo to yield $28 \mathrm{mg}(100 \%)$ of the crude product $\mathbf{2 1}$ as a colorless oil. The product was used crude in the next step without further purification: IR (neat) 2927, 2854, 1720, 1611, 1512, $1251,1201,1097,841 \mathrm{~cm}^{-1 ;}{ }_{1} \mathrm{H}$ NMR $\left(\mathrm{CDCl}_{3}, 400 \mathrm{MHz}\right) \delta 9.74(\mathrm{q}, J=7.7 \mathrm{~Hz}, 1 \mathrm{H}), 7.32(\mathrm{~m}$, $8 \mathrm{H}), 7.12(\mathrm{t}, J=7.7 \mathrm{~Hz}, 4 \mathrm{H}), 6.84(\mathrm{~d}, J=8.8 \mathrm{~Hz}, 2 \mathrm{H}), 5.36(\mathrm{q}, J=1.4 \mathrm{~Hz}, 1 \mathrm{H}), 5.10(\mathrm{~s}, 1 \mathrm{H})$, $4.69(\mathrm{dd}, J=2.2,9.16 \mathrm{~Hz}, 1 \mathrm{H}), 4.50(\mathrm{~s}, 2 \mathrm{H}), 3.95(\mathrm{~s}, 2 \mathrm{H}), 3.78(\mathrm{~s}, 3 \mathrm{H}), 3.76(\mathrm{~s}, 1 \mathrm{H}), 3.23(\mathrm{~d}$, $J=12.1 \mathrm{~Hz}, 1 \mathrm{H}), 3.18(\mathrm{~d}, J=14.0 \mathrm{~Hz}, 1 \mathrm{H}), 2.86(\mathrm{~d}, J=13.2 \mathrm{~Hz}, 2 \mathrm{H}), 2.74(\mathrm{dd}, J=1.5,15.8$ $\mathrm{Hz}, 2 \mathrm{H}), 2.53(\mathrm{~m}, 2 \mathrm{H}), 1.7$ (bs, 1H), $1.55(\mathrm{bs}, 1 \mathrm{H}), 0.77(\mathrm{~s}, 9 \mathrm{H}),-0.08(\mathrm{~s}, 3 \mathrm{H}),-0.10(\mathrm{~s}$, $3 \mathrm{H}) ;{ }^{13} \mathrm{C} \mathrm{NMR}\left(\mathrm{CDCl}_{3}, 100 \mathrm{MHz}\right) \delta 205.9,159.0,151.1,141.3,138.4,130.5,130.3,129.8$, $129.8,128.57,128.0,127.9,127.8,126.1,122.1,114.6,74.0,72.6,71.3,70.6,64.3,55.6,45.1$, 41.2, 39.7, 29.9, 25.9, 18.1, -4.8, -5.5; HRMS $(\mathrm{M}+\mathrm{H})^{+}$calcd for $\mathrm{C}_{40} \mathrm{H}_{51} \mathrm{NO}_{7} \mathrm{Si}+\mathrm{H}: 685.3435$, found 685.3411 .

\section{2-(4-Methoxybenzyl)-4-oxo-6-[4-(trimethylsilanyl)but-3-ynyl]-3,4-dihydro-2H-pyridine-1- carboxylic acid phenyl ester (28)}

Magnesium turnings $(2.16 \mathrm{~g}, 889 \mathrm{mmol})$ were mechanically activated by stirring at rt overnight under argon and then $40 \mathrm{~mL}$ of anhydrous ether was added. A portion of 4-bromo-1trimethylsilyl-1-butyne $(3.65 \mathrm{~g}, 178 \mathrm{mmol})$ in $20 \mathrm{~mL}$ of anhydrous ether was slowly added to the mixture. Once the reaction was initiated, the mixture was cooled to $10{ }^{\circ} \mathrm{C}$, and the remaining bromide was continuously added over a period of $2 \mathrm{~h}$. After the addition was complete, the reaction mixture was stirred at $10{ }^{\circ} \mathrm{C}$ for $3 \mathrm{~h}$, warmed up to rt, and stirred for an additional 4 $\mathrm{h}$ to form the Grignard reagent 26 . In a separate flask, copper bromide dimethyl sulfide complex $(6.09 \mathrm{~g}, 296 \mathrm{mmol})$ was mixed with $20 \mathrm{~mL}$ of freshly distilled THF under argon and cooled to $-78{ }^{\circ} \mathrm{C}$. To a solution of 2-(4-methoxybenzyl)-4-oxo-3,4-dihydro- $2 \mathrm{H}$-pyridine-1- carboxylic acid phenyl ester (11) $(2.00 \mathrm{~g}, 593 \mathrm{mmol})$ in THF $(10 \mathrm{~mL})$ was added a premixed solution of TMSCl $(8.30 \mathrm{~mL}, 652 \mathrm{mmol})$ and $\mathrm{Et}_{3} \mathrm{~N}(9.5 \mathrm{~mL}, 682 \mathrm{mmol})$. A solution of the above prepared Grignard reagent 26 in $\mathrm{Et}_{2} \mathrm{O}$ (diluted with $50 \mathrm{~mL}$ of THF) was added dropwise over a period of $1 \mathrm{~h}$. Once the addition was complete, the resulting slurry was stirred at $-78^{\circ} \mathrm{C}$ for $2 \mathrm{~h}$ and then warmed to $-45^{\circ} \mathrm{C}$. After $24 \mathrm{~h}$, the reaction mixture was quenched with a carefully buffered solution ( $\mathrm{pH} 8)$ of $\mathrm{NH}_{4} \mathrm{OH}$ and $\mathrm{NH}_{4} \mathrm{Cl}(100 \mathrm{~mL})$ and the phases were separated. The aqueous phase was extracted with $\mathrm{Et}_{2} \mathrm{O}(3 \times 50 \mathrm{~mL})$, and the combined organic layers were washed with $10 \% \mathrm{NH}_{4} \mathrm{OH}$ until no further blue color was observed in the aqueous layer. After drying over anhydrous $\mathrm{K}_{2} \mathrm{CO}_{3}$ and filtering through Celite, the solvent was removed under reduced 
pressure to give $3.4 \mathrm{~g}$ of crude $\mathbf{2 7}$, which was dissolved in $10 \mathrm{~mL}$ of anhydrous DMSO. In a separate flask, IBX (9.96 g, $356 \mathrm{mmol})$ and MPO (4.45 g, $356 \mathrm{mmol})$ were dissolved in $20 \mathrm{~mL}$ of DMSO at $10^{\circ} \mathrm{C}$. The IBX/MPO mixture was stirred until complete dissolution and was then added in one portion at $0{ }^{\circ} \mathrm{C}$ to the crude silyl enol ether 27 solution. The mixture was stirred vigorously at rt overnight. The reaction mixture was carefully diluted with $5 \% \mathrm{NaHCO}_{3}(50$ $\mathrm{mL})$ and extracted with $\mathrm{Et}_{2} \mathrm{O}(3 \times 100 \mathrm{~mL})$. The combined organic phase was dried over anhydrous $\mathrm{MgSO}_{4}$, filtered through Celite, and the solvent was removed in vacuo to yield the crude product 28. Purification by silica gel chromatography (10 to 25\% EtOAc in hexanes) gave $2.3 \mathrm{~g}$ (55\%, 2 steps) of $\mathbf{2 8}$ as a yellow foam: IR (neat) 2955, 2171, 1725, 1666, 1513,

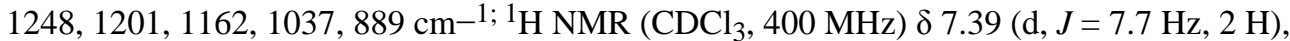
$7.27(\mathrm{~d}, J=7.0 \mathrm{~Hz}, 1 \mathrm{H}), 7.15(\mathrm{dd}, J=1.8,6.6 \mathrm{~Hz}, 2 \mathrm{H}), 6.93(\mathrm{~d}, J=7.3 \mathrm{~Hz}, 2 \mathrm{H}), 6.82(\mathrm{dd}$, $J=1.8,6.2 \mathrm{~Hz}, 2 \mathrm{H}), 5.66(\mathrm{~s}, 1 \mathrm{H}), 5.10(\mathrm{dq}, J=1.5,6.2 \mathrm{~Hz}, 1 \mathrm{H}), 3.79(\mathrm{~s}, 3 \mathrm{H}), 3.29(\mathrm{~m}, 1 \mathrm{H})$, $3.10(\mathrm{dd}, J=7.3,7.3 \mathrm{~Hz}, 1 \mathrm{H}), 2.89$ (dd, $J=8.1,6.2 \mathrm{~Hz}, 1 \mathrm{H}), 2.84(\mathrm{~d}, J=5.9 \mathrm{~Hz}, 1 \mathrm{H}), 2.71$ $(\mathrm{m}, 1 \mathrm{H}), 2.55(\mathrm{t}, J=6.6 \mathrm{~Hz}, 2 \mathrm{H}), 2.46(\mathrm{~d}, J=17.2 \mathrm{~Hz}, 1 \mathrm{H}), 0.11(\mathrm{~s}, 9 \mathrm{H}) ;{ }^{13} \mathrm{C} \mathrm{NMR}$ $\left(\mathrm{CDCl}_{3}, 100 \mathrm{MHz}\right) \delta 193.2,158.8,155.4,151.8,150.4,130.8,129.6,129.2,126.5,121.6$ $(122.0 \mathrm{r}), 114.7,114.3,105.0,87.3,58.5,55.5,40.3,36.1,35.0,19.1,0.2$; HRMS $(\mathrm{M}+\mathrm{H})^{+}$ calcd for $\mathrm{C}_{27} \mathrm{H}_{31} \mathrm{NO}_{4} \mathrm{Si}+\mathrm{H}: 462.2101$, found 462.2085 .

\section{6-(3-Bromobut-3-enyl)-2-(4-methoxybenzyl)-4-oxo-3,4-dihydro-2H-pyridine-1-carboxylic acid phenyl ester (29)}

A solution of $28(1.43 \mathrm{~g}, 3.10 \mathrm{mmol})$ in THF $(35 \mathrm{~mL})$ was cooled to $-78{ }^{\circ} \mathrm{C}$.

Tetrabutylammonium fluoride (1.0 M in THF, $3.72 \mathrm{~mL}, 3.72 \mathrm{mmol}$ ) was added dropwise over a period of $10 \mathrm{~min}$. After the addition was complete, the reaction mixture was stirred at $-78^{\circ}$ $\mathrm{C}$ for $30 \mathrm{~min}$, warmed to $\mathrm{rt}$, and stirred for an additional $1 \mathrm{~h}$ at rt. The reaction mixture was poured into saturated aqueous $\mathrm{NH}_{4} \mathrm{Cl}(100 \mathrm{~mL})$ and then extracted with $\mathrm{Et}_{2} \mathrm{O}(3 \times 250 \mathrm{~mL})$. The combined organic extracts were dried over $\mathrm{MgSO}_{4}$, filtered through Celite, and concentrated in vacuo to yield the crude acetylene product. Purification by silica gel chromatography ( 10 to $25 \%$ EtOAc in hexanes) gave $1.11 \mathrm{~g} \mathrm{(93 \% )} \mathrm{of} \mathrm{the} \mathrm{desired} \mathrm{acetylene} \mathrm{as}$ a colorless oil which was used directly in the next step. IR (neat) 3286, 2932, 1738, 1665, 1598, $1512,1402,1333,1301,1247,1162,1040,829,749,689 \mathrm{~cm}^{-1 ;}{ }_{1} \mathrm{H}$ NMR $\left(\mathrm{CDCl}_{3}, 100 \mathrm{MHz}\right)$ $\delta 7.39(\mathrm{t}, J=8.0 \mathrm{~Hz}, 2 \mathrm{H}), 7.26(\mathrm{~m}, 1 \mathrm{H}), 7.13(\mathrm{~d}, J=8.4 \mathrm{~Hz}, 2 \mathrm{H}), 6.94(\mathrm{~d}, J=7.6 \mathrm{~Hz}, 2 \mathrm{H})$, $6.84(\mathrm{~d}, J=8.4 \mathrm{~Hz}, 2 \mathrm{H}), 5.68(\mathrm{~s}, 1 \mathrm{H}), 5.11(\mathrm{q}, J=7.6 \mathrm{~Hz}, 1 \mathrm{H}), 3.79(\mathrm{~s}, 3 \mathrm{H}), 3.27(\mathrm{~m}, 1 \mathrm{H})$, $3.10(\mathrm{dd}, J=7.2,6.4 \mathrm{~Hz}, 1 \mathrm{H}), 2.90(\mathrm{dd}, J=8.0,5.6 \mathrm{~Hz}, 1 \mathrm{H}), 2.87(\mathrm{dd}, J=6.0,11.2 \mathrm{~Hz}, 1 \mathrm{H})$, $2.76(\mathrm{~m}, 1 \mathrm{H}), 2.52(\mathrm{~m}, 2 \mathrm{H}), 2.45(\mathrm{~s}, 1 \mathrm{H}), 2.07(\mathrm{t}, J=2.0 \mathrm{~Hz}, 1 \mathrm{H}) ;{ }^{13} \mathrm{C} \mathrm{NMR}\left(\mathrm{CDCl}_{3}, 100\right.$ MHz) $\delta 193.2,158.8,155.3,151.7,150.4,130.7,129.7,129.1,128.5,126.5,121.5$ (121.9 r), $114.3\left(114.6\right.$ r), 82.4 , 7.6, 58.3, 55.5, 40.2, 36.0, 34.7, 17.7; HRMS $(\mathrm{M}+\mathrm{H})^{+}$calcd for $\mathrm{C}_{24} \mathrm{H}_{23} \mathrm{NO}_{4}+\mathrm{H}: 390.1705$, found 390.1711 .

A solution of the above acetylene $(44.0 \mathrm{mg}, 0.113 \mathrm{mmol})$ in $\mathrm{CH}_{2} \mathrm{Cl}_{2}(1.0 \mathrm{~mL})$ at $0{ }^{\circ} \mathrm{C}$ under

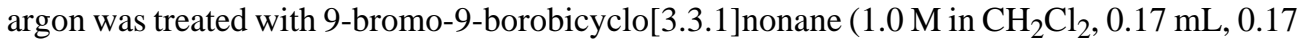
$\mathrm{mmol}$ ). The resulting mixture was allowed to warm to $\mathrm{rt}$ and stirred for $24 \mathrm{~h}$. After this time, the mixture was cooled to $0{ }^{\circ} \mathrm{C}$ and ethanolamine $(1.1 \mathrm{~mL})$ was added, followed by $\mathrm{MeOH}$ $(2.5 \mathrm{~mL})$. The mixture was then diluted with $\mathrm{Et}_{2} \mathrm{O}(15 \mathrm{~mL})$ and shaken with saturated aqueous potassium sodium tartrate $(15 \mathrm{~mL})$. The aqueous phase was extracted with $\mathrm{Et}_{2} \mathrm{O}(2 \times 15 \mathrm{~mL})$, and the combined organic extracts were dried over $\mathrm{MgSO}_{4}$, filtered through Celite, and concentrated in vacuo to yield the crude product 29. Purification by silica gel chromatography (10-15\% EtOAc in hexanes) gave $45.0 \mathrm{mg}$ (85\%) of $\mathbf{2 9}$ as a colorless oil: IR (neat) 2927, 1736, $1666,1597,1513,1406,1301,1248,1202,1164,1035,890 \mathrm{~cm}^{-1 ;}{ }_{1} \mathrm{H} \mathrm{NMR}\left(\mathrm{CDCl}_{3}, 400 \mathrm{MHz}\right)$ $\delta 7.38(\mathrm{dt}, J=2.0,8.8 \mathrm{~Hz}, 2 \mathrm{H}), 7.27(\mathrm{t}, J=1.2 \mathrm{~Hz}, 1 \mathrm{H}), 7.08(\mathrm{dd}, J=2.8,8.8 \mathrm{~Hz}, 2 \mathrm{H}), 6.97$ $(\mathrm{dd}, J=2.0,2.8 \mathrm{~Hz}, 2 \mathrm{H}), 6.84(\mathrm{dd}, J=2.8,8.8 \mathrm{~Hz}, 2 \mathrm{H}), 5.60(\mathrm{dd}, J=0.8,1.6 \mathrm{~Hz}, 2 \mathrm{H}), 5.44$ $(\mathrm{d}, J=2.4 \mathrm{~Hz}, 1 \mathrm{H}), 5.07(\mathrm{dq}, J=2.0,8.0 \mathrm{~Hz}, 1 \mathrm{H}), 3.78(\mathrm{~s}, 3 \mathrm{H}), 3.20(\mathrm{~m}, 1 \mathrm{H}), 3.04(\mathrm{dd}, J=$ $8.8,9.2 \mathrm{~Hz}, 1 \mathrm{H}), 2.81(\mathrm{~m}, 4 \mathrm{H}), 2.45(\mathrm{dt}, J=1.6,17.2 \mathrm{~Hz}, 2 \mathrm{H}) ;{ }^{13} \mathrm{C} \mathrm{NMR}\left(\mathrm{CDCl}_{3}, 100 \mathrm{MHz}\right)$ 
$\delta 192.9,158.7,155.8,151.6,150.3,132.2,130.6,129.7,128.8,126.5,121.4,118.3,114.3$, 114.0, 58.3, 55.6, 40.34, 40.0, 36.1, 34.4; HRMS $(\mathrm{M}+\mathrm{H})^{+}$calcd for $\mathrm{C}_{24} \mathrm{H}_{24} \mathrm{BrNO}_{4}+\mathrm{H}$ : 470.0967, found 470.0959 .

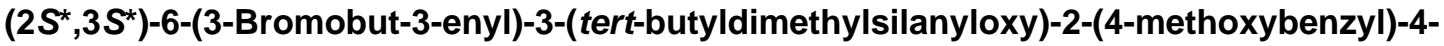 oxo-3,4-dihydro-2 $\mathrm{H}$-pyridine-1-carboxylic acid phenyl ester (25)}

To a stirred solution of 29 (224 $\mathrm{mg}, 0.477 \mathrm{mmol})$ in $5 \mathrm{~mL}$ of toluene at $\mathrm{rt}$ was added lead (IV) acetate $(275 \mathrm{mg}, 0.620 \mathrm{mmol})$. The resulting mixture was refluxed for $3 \mathrm{~h}$, cooled to rt, and additional lead (IV) acetate $(275 \mathrm{mg}, 0.620 \mathrm{mmol}$ ) was added. The mixture refluxed for $3 \mathrm{~h}$. After cooling to rt, the solution was filtered through Celite with $\mathrm{CH}_{2} \mathrm{Cl}_{2}$. The filtrate was washed with saturated aqueous $\mathrm{NaHCO}_{3}(40 \mathrm{~mL})$, and the aqueous phase was extracted with $\mathrm{CH}_{2} \mathrm{Cl}_{2}(3 \times 40 \mathrm{~mL})$. The combined organic phase was dried over $\mathrm{Na}_{2} \mathrm{SO}_{4}$, filtered through Celite, and concentrated in vacuo to yield the crude acetate. Purification by silica gel chromatography (20 to $30 \%$ EtOAc in hexanes) gave $173 \mathrm{mg}(69 \%)$ of the desired product as a colorless oil which was used directly in the next step. IR (neat) 2926, 1743, 1678, 1591, 1514, $1248,1219,1182,1032,839 \mathrm{~cm}^{-1 ;}{ }_{1} \mathrm{H}$ NMR $\left(\mathrm{CDCl}_{3}, 400 \mathrm{MHz}\right) \delta 7.38(\mathrm{dt}, J=1.6,7.2 \mathrm{~Hz}, 2$ H), $7.12(\mathrm{dd}, J=2.4,6.8 \mathrm{~Hz}, 2 \mathrm{H}), 6.84(\mathrm{~m}, 5 \mathrm{H}), 5.70(\mathrm{~s}, 1 \mathrm{H}), 5.62(\mathrm{~m}, 1 \mathrm{H}), 5.47(\mathrm{~d}, J=2.0$ $\mathrm{Hz}, 1 \mathrm{H}), 5.25(\mathrm{dt}, J=2.8,7.2 \mathrm{~Hz}, 1 \mathrm{H}), 4.94(\mathrm{t}, J=2.0 \mathrm{~Hz}, 1 \mathrm{H}), 3.20(\mathrm{~m}, 1 \mathrm{H}), 3.00(\mathrm{dd}, J=$ 8.8, $8.8 \mathrm{~Hz}, 1 \mathrm{H}), 2.93(\mathrm{~m}, 4 \mathrm{H}), 2.76(\mathrm{~m}, 1 \mathrm{H}), 2.65(\mathrm{~m}, 2 \mathrm{H}), 2.11(\mathrm{~s}, 3 \mathrm{H}) ;{ }^{13} \mathrm{C}$ NMR $\left(\mathrm{CDCl}_{3}, 100 \mathrm{MHz}\right) \delta 187.6,169.6,159.1,157.6,150.2$ (152.0 r), 132.1, 130.7, 130.5, 129.9, 127.6, 126.7, 121.3, 118.4, 114.5, 112.6, 71.3, 61.9, 55.5, 40.1, 34.5, 33.5, 21.0; HRMS (M $+\mathrm{H})^{+}$calcd for $\mathrm{C}_{26} \mathrm{H}_{26} \mathrm{BrNO}_{6}+\mathrm{H}: 528.1022$, found 528.1022.

To a solution of the above acetate (390 mg, $0.734 \mathrm{mmol})$ in $\mathrm{H}_{2} \mathrm{O}: \mathrm{MeOH}(20 \mathrm{~mL}, 1: 4)$ was added $\mathrm{ScOTf}_{3}(20 \mathrm{~mol} \%, 72.0 \mathrm{mg}, 0.147 \mathrm{mmol})$. The resulting mixture was stirred at $60{ }^{\circ} \mathrm{C}$ for $24 \mathrm{~h}$. The reaction mixture was cooled to rt, poured into $\mathrm{H}_{2} \mathrm{O}(20 \mathrm{~mL})$, and extracted with $\mathrm{CH}_{2} \mathrm{Cl}_{2}(3 \times 20 \mathrm{~mL})$. The combined organic extracts were dried over $\mathrm{Na}_{2} \mathrm{SO}_{4}$, filtered through Celite with $\mathrm{CH}_{2} \mathrm{Cl}_{2}$, and the solvent was removed in vacuo to yield the crude product.

Purification by silica gel chromatography (20 to $30 \%$ EtOAc in hexanes) gave $355 \mathrm{mg}$ (100 $\%$ ) of the desired alcohol as a white foam which was used directly in the next step. IR (neat) $3376,2919,1738,1666,1589,1513,1248,1179,838 \mathrm{~cm}^{-1 ; 1} \mathrm{H} \mathrm{NMR}\left(\mathrm{CDCl}_{3}, 400 \mathrm{MHz}\right) \delta$ $7.39(\mathrm{t}, J=8.0 \mathrm{~Hz}, 2 \mathrm{H}), 7.26(\mathrm{t}, J=7.2 \mathrm{~Hz}, 1 \mathrm{H}), 7.10(\mathrm{~d}, J=8.8 \mathrm{~Hz}, 2 \mathrm{H}), 7.00(\mathrm{~d}, J=7.6$ $\mathrm{Hz}, 2 \mathrm{H}), 6.85(\mathrm{~d}, J=8.4 \mathrm{~Hz}, 2 \mathrm{H}), 5.62(\mathrm{~d}, J=0.8 \mathrm{~Hz}, 1 \mathrm{H}), 5.60(\mathrm{~s}, 1 \mathrm{H}), 5.45(\mathrm{~d}, J=1.2 \mathrm{~Hz}$, $1 \mathrm{H}), 5.11(\mathrm{dt}, J=1.6,8.0 \mathrm{~Hz}, 1 \mathrm{H}), 3.79(\mathrm{~s}, 4 \mathrm{H}), 3.20(\mathrm{~m}, 1 \mathrm{H}), 2.97(\mathrm{~m}, 6 \mathrm{H}) ;{ }^{13} \mathrm{C} \mathrm{NMR}$ $\left(\mathrm{CDCl}_{3}, 100 \mathrm{MHz}\right) \delta 192.3,159.0,157.3,152.3,150.5,132.2,130.6,129.8,128.2,126.6$, 121.6, 118.4, 114.4, 111.1, 70.9, 64.1, 55.5, 40.2, 34.5, 38.8; HRMS $(\mathrm{M}+\mathrm{H})^{+}$calcd for $\mathrm{C}_{24} \mathrm{H}_{24} \mathrm{BrNO}_{5}+\mathrm{H}: 486.0916$, found 486.0911 .

To a solution of the above alcohol $(355 \mathrm{mg}, 0.733 \mathrm{mmol})$ in DMF $(10 \mathrm{~mL})$ at $20^{\circ} \mathrm{C}$ was added imidazole ( $100 \mathrm{mg}, 1.47 \mathrm{mmol})$ and tert-butyldimethylsilyl chloride $(166 \mathrm{mg}, 1.10 \mathrm{mmol})$. The resulting mixture was stirred at $\mathrm{rt}$ for $24 \mathrm{~h}$. The reaction mixture was quenched with saturated aqueous $\mathrm{NaHCO}_{3}(50 \mathrm{~mL})$ and extracted with $\mathrm{CH}_{2} \mathrm{Cl}_{2}(3 \times 50 \mathrm{~mL})$. The combined organic layers were washed with brine $(30 \mathrm{~mL})$, dried over $\mathrm{Na}_{2} \mathrm{SO}_{4}$, filtered through Celite with $\mathrm{CH}_{2} \mathrm{Cl}_{2}$, and the solvent was removed in vacuo to yield the crude TBS ether 25 . Purification by silica gel chromatography (30 to $40 \%$ EtOAc in hexanes) gave $397 \mathrm{mg}(90 \%)$ of $\mathbf{2 5}$ as a colorless oil: IR (neat) 2929, 2856, 1737, 1676, 1598, 1513, 1249, 1182, $841 \mathrm{~cm}^{-1 ; 1} \mathrm{H}$ NMR $\left(\mathrm{CDCl}_{3}, 400 \mathrm{MHz}\right) \delta 7.38(\mathrm{dt}, J=1.6,7.2 \mathrm{~Hz}, 2 \mathrm{H}), 7.26(\mathrm{~m}, 1 \mathrm{H}), 7.10(\mathrm{dd}, J=2.0,6.8 \mathrm{~Hz}$, $2 \mathrm{H}), 6.94(\mathrm{dd}, J=1.2,7.5 \mathrm{~Hz}, 2 \mathrm{H}), 6.86(\mathrm{dd}, J=2.0,6.4 \mathrm{~Hz}, 2 \mathrm{H}), 5.60(\mathrm{~m}, 1 \mathrm{H}), 5.55(\mathrm{~s}, 1$ H), $5.44(\mathrm{~d}, J=1.6 \mathrm{~Hz}, 1 \mathrm{H}), 4.98(\mathrm{dt}, J=1.6,8.0 \mathrm{~Hz}, 1 \mathrm{H}), 3.80(\mathrm{~s}, 3 \mathrm{H}), 3.70(\mathrm{~d}, J=1.2 \mathrm{~Hz}$, $1 \mathrm{H}), 3.21(\mathrm{~m}, 1 \mathrm{H}), 2.79(\mathrm{~m}, 5 \mathrm{H}), 0.84(\mathrm{~s}, 9 \mathrm{H}), 0.04(\mathrm{~s}, 3 \mathrm{H}), 0.02(\mathrm{~s}, 3 \mathrm{H}) ;{ }^{13} \mathrm{C} \mathrm{NMR}$ $\left(\mathrm{CDCl}_{3}, 100 \mathrm{MHz}\right) \delta 191.7,158.9,155.9,152.5,150.5,132.4,130.5,129.8,128.5,126.4$, 
121.5, 118.3, 114.3, 111.6, 71.5, 65.6, 55.5, 40.3, 34.3, 33.2, 25.8, 18.2, -4.6, -5.0; HRMS

$(\mathrm{M}+\mathrm{H})^{+}$calcd for $\mathrm{C}_{30} \mathrm{H}_{38} \mathrm{BrNO}_{5} \mathrm{Si}+\mathrm{H}: 600.1781$, found 600.1800 .

$\left(2 R^{\star}, 5 S^{\star}, 6 S^{\star}\right)-2-(2-B e n z y l o x y m e t h y l a l l y l)-2-(3-b r o m o b u t-3-e n y l)-5-(t e r t-$ butyldimethylsilanyloxy)- 6-(4-methoxybenzyl)-4-oxo-piperidine-1-carboxylic acid phenyl ester (31)

A well-stirred solution of dihydropyridone $25(57 \mathrm{mg}, 0.097 \mathrm{mmol})$ in $3 \mathrm{~mL}$ of dry $\mathrm{CH}_{2} \mathrm{Cl}_{2}$ was cooled to $-78{ }^{\circ} \mathrm{C}$. TMSOTf $(40 \mu \mathrm{l}, 0.22 \mathrm{mmol})$ was added in one portion, giving rise to a deep orange color. After 5 min, neat (2-benzyloxymethylallyl)tributylstannane reagent 6 (76 $\mathrm{mg}, 0.15 \mathrm{mmol}$ ) was added dropwise over a 10 -min period. The reaction mixture was stirred for $4 \mathrm{~h}$ at $-78{ }^{\circ} \mathrm{C}$, warmed to $-45^{\circ} \mathrm{C}$, and stirred for additional $6 \mathrm{~h}$. The reaction mixture was quenched with saturated aqueous $\mathrm{NaHCO}_{3}(20 \mathrm{~mL})$ and allowed to warm up to rt, during which time it became colorless. The mixture was extracted with $\mathrm{CH}_{2} \mathrm{Cl}_{2}(3 \times 20 \mathrm{~mL})$. The combined organic extracts were dried over $\mathrm{Na}_{2} \mathrm{SO}_{4}$ and filtered through Celite. The solvent was removed in vacuo to yield $110 \mathrm{mg}$ of the crude silyl enol ether product 30, which was dissolved in wet $\mathrm{MeOH}(5 \mathrm{~mL})$. Silica gel $(100 \mathrm{mg})$ was added, followed by $\mathrm{H}_{2} \mathrm{O}(0.5 \mathrm{~mL})$ and oxalic acid $(20$ $\mathrm{mg}, 0.22 \mathrm{mmol}$ ). The reaction mixture was stirred at $\mathrm{rt}$ for $16 \mathrm{~h}$. The reaction mixture was filtered and then extracted with $\mathrm{CH}_{2} \mathrm{Cl}_{2}(3 \times 20 \mathrm{~mL})$. The combined organic extracts were dried over $\mathrm{Na}_{2} \mathrm{SO}_{4}$, filtered through Celite, and the solvent was removed in vacuo to yield the crude piperidone product 31. Purification by silica gel chromatography ( 0 to $20 \%$ EtOAc in hexanes) gave $62 \mathrm{mg}(85 \%)$ of $\mathbf{3 1}$ as a colorless oil: IR (neat) 2926, 2853, 1722, 1512, 1379, 1250, 1199, $1072,844 \mathrm{~cm}^{-1 ;}{ }_{1} \mathrm{H}$ NMR $\left(\mathrm{C}_{6} \mathrm{D}_{6}, 400 \mathrm{MHz}\right) \delta 7.20(\mathrm{~m}, 10 \mathrm{H}), 6.89(\mathrm{t}, J=7.6 \mathrm{~Hz}, 2 \mathrm{H}), 6.67$ $(\mathrm{d}, J=7.6 \mathrm{~Hz}, 2 \mathrm{H}), 5.38(\mathrm{~s}, 1 \mathrm{H}), 5.26(\mathrm{~m}, 3 \mathrm{H}), 4.84(\mathrm{~m}, 1 \mathrm{H}), 4.31(\mathrm{~s}, 2 \mathrm{H}), 3.92(\mathrm{~s}, 1 \mathrm{H}), 3.90$ (s, 2H), $3.50(\mathrm{~d}, J=17.2 \mathrm{~Hz}, 1 \mathrm{H}), 3.27(\mathrm{~s}, 1 \mathrm{H}), 3.25(\mathrm{~s}, 3 \mathrm{H}), 3.07(\mathrm{dd}, J=3.2,13.6 \mathrm{~Hz}, 1 \mathrm{H})$, $2.84(\mathrm{~d}, J=17.2 \mathrm{~Hz}, 1 \mathrm{H}), 2.54(\mathrm{~m}, 2 \mathrm{H}), 2.24(\mathrm{~m}, 2 \mathrm{H}), 1.72(\mathrm{~m}, 1 \mathrm{H}), 1.54(\mathrm{~m}, 1 \mathrm{H}), 0.90(\mathrm{~s}, 9$ $\mathrm{H}), 0.00(\mathrm{~s}, 3 \mathrm{H}),-0.20(\mathrm{~s}, 3 \mathrm{H}) ;{ }^{13} \mathrm{C}$ NMR $\left(\mathrm{C}_{6} \mathrm{D}_{6}, 100 \mathrm{MHz}\right) \delta 205.6,159.1,151.7,142.0$, $138.8,133.7,130.5,129.7,129.5,128.4,128.2,127.9,127.7,127.5,127.1,125.5,122.1,117.2$, $74.1,72.2,70.9,64.5,62.5,54.7,45.2,41.3,37.5,37.4,25.7,25.5,18.2,-4.9,-5.7$; HRMS $(\mathrm{M}+\mathrm{H})^{+}$calcd for $\mathrm{C}_{41} \mathrm{H}_{52} \mathrm{NO}_{6} \mathrm{BrSi}+\mathrm{H}: 761.2747$, found 761.2709 .

\section{$\left(2 R^{\star}, 4 S^{\star}, 5 S^{\star}, 6 S^{\star}\right)-2-(2-B e n z y l o x y m e t h y l a l l y l)-2-(3-b r o m o b u t-3-e n y l)-4,5-d i h y d r o x y-6-(4-$ methoxybenzyl)piperidine-1-carboxylic acid phenyl ester (32)}

A solution of $\mathbf{3 1}(57.0 \mathrm{mg}, 0.0758 \mathrm{mmol})$ in THF $(4 \mathrm{~mL})$ was cooled to $-40{ }^{\circ} \mathrm{C}$.

Tetrabutylammonium fluoride (1.0 M in THF, $91 \mu 1,0.091 \mathrm{mmol})$ was added dropwise over a period of $5 \mathrm{~min}$. After the addition was complete, the reaction mixture was stirred at $-40{ }^{\circ} \mathrm{C}$ for $1 \mathrm{~h}$, warmed to $-20^{\circ} \mathrm{C}$, and stirred for an additional 2 hours. The reaction mixture was poured into saturated aqueous $\mathrm{NH}_{4} \mathrm{Cl}(10 \mathrm{~mL})$ and then extracted with $\mathrm{CH}_{2} \mathrm{Cl}_{2}(3 \times 10 \mathrm{~mL})$. The combined organic extracts were dried over $\mathrm{Na}_{2} \mathrm{SO}_{4}$, filtered through Celite, and concentrated in vacuo to yield the crude alcohol. To a solution of tetramethylammonium triacetoxyborohydride $(80.0 \mathrm{mg}, 0.303 \mathrm{mmol})$, in $4.0 \mathrm{~mL}$ of freshly distilled acetone at $\mathrm{rt}$, was added acetic acid $(35.0 \mu 1,0.607 \mathrm{mmol})$. After stirring for $15 \mathrm{~min}$, the above crude alcohol compound in $4.0 \mathrm{~mL}$ of freshly distilled acetone was added dropwise over a period of $5 \mathrm{~min}$. The reaction mixture was stirred at $\mathrm{rt}$ for $16 \mathrm{~h}$. The reaction mixture was quenched with saturated aqueous ammonium chloride $(25 \mathrm{~mL})$, and one half of the acetone was removed in vacuo. The solution was extracted with $\mathrm{CH}_{2} \mathrm{Cl}_{2}(3 \times 25 \mathrm{~mL})$. The combined organic extracts were washed with brine $(25 \mathrm{~mL})$, dried over $\mathrm{Na}_{2} \mathrm{SO}_{4}$, filtered through Celite, and the solvent was removed in vacuo to yield the crude product. Purification by silica gel chromatography ( 30 to $50 \%$ EtOAc in hexanes) gave $45 \mathrm{mg}$ ( $92 \%$ over 2 steps) of $\mathbf{3 2}$ as a colorless oil: IR (neat) $3456,2924,1693,1512,1454,1248,1203,1034 \mathrm{~cm}^{-1 ; 1} \mathrm{H} \mathrm{NMR}\left(\mathrm{CDCl}_{3}, 400 \mathrm{MHz}\right) \delta 7.40$ $(\mathrm{t}, J=7.6 \mathrm{~Hz}, 1 \mathrm{H}), 7.28(\mathrm{~m}, 11 \mathrm{H}), 7.14(\mathrm{dd}, J=1.6,7.2 \mathrm{~Hz}, 2 \mathrm{H}), 5.58(\mathrm{~d}, J=1.6 \mathrm{~Hz}, 1 \mathrm{H}), 5.40$ $(\mathrm{d}, J=2.0 \mathrm{~Hz}, 1 \mathrm{H}), 5.33(\mathrm{~d}, J=1.2 \mathrm{~Hz}, 1 \mathrm{H}), 5.16(\mathrm{~s}, 1 \mathrm{H}), 4.50(\mathrm{dd}, J=11.6,6.0 \mathrm{~Hz}, 2 \mathrm{H}), 4.42$ (bd, $J=12.0 \mathrm{~Hz}, 1 \mathrm{H}), 4.14$ (bs, 1H), 4.04 (dd, $J=12.8,8.0 \mathrm{~Hz}, 2 \mathrm{H}), 3.79$ (s, 4H), 3.12 (dd, 
$J=2.8,12.8 \mathrm{~Hz}, 1 \mathrm{H}), 3.02(\mathrm{dd}, J=14.0,6.8 \mathrm{~Hz}, 2 \mathrm{H}), 2.83(\mathrm{t}, J=12.4 \mathrm{~Hz}, 1 \mathrm{H}), 2.73(\mathrm{~m}, 1 \mathrm{H})$, $2.48(\mathrm{~m}, 2 \mathrm{H}), 2.14(\mathrm{dd}, J=6.0,14.8 \mathrm{~Hz}, 1 \mathrm{H}), 1.95(\mathrm{~m}, 2 \mathrm{H}), 1.83(\mathrm{~m}, 1 \mathrm{H}), 1.63(\mathrm{~d}, J=3.2 \mathrm{~Hz}$, $1 \mathrm{H}) ;{ }^{13} \mathrm{C} \mathrm{NMR}\left(\mathrm{CDCl}_{3}, 100 \mathrm{MHz}\right) \delta 158.6,154.6,151.2,142.4,138.2,133.9,130.9,130.2$, $129.7,128.65,128.1,128.0,125.8,122.1,118.0,117.2,114.3,74.4,74.3,72.4,69.7,64.2$, 61.2, 55.5, 41.5, 40.0, 38.0, 37.4, 36.8; $\mathrm{HRMS}(\mathrm{M}+\mathrm{H})^{+}$calcd for $\mathrm{C}_{35} \mathrm{H}_{40} \mathrm{BrNO}_{6}+\mathrm{H}: 650.2117$, found 650.2101 .

$\left(2 R^{\star}, 4 S^{\star}, 5 S^{\star}, 6 S^{\star}\right)-4-B e n z o y l o x y-2-(2-b e n z y l o x y m e t h y l a l l y l)-2-(3-b r o m o b u t-3-e n y l)-5-$ hydroxy-6-(4-methoxybenzyl)piperidine-1-carboxylic acid phenyl ester (33)

A solution of $32(23.9 \mathrm{mg}, 36.9 \mu \mathrm{mol})$ in $\mathrm{CH}_{2} \mathrm{Cl}_{2}(1.5 \mathrm{~mL})$ was cooled to $-20{ }^{\circ} \mathrm{C}$. $\mathrm{Et}_{3} \mathrm{~N}(6.0$ $\mu \mathrm{L}, 41 \mu \mathrm{mol})$ was added dropwise, followed by a catalytic amount of DMAP. The reaction mixture was stirred at $-20{ }^{\circ} \mathrm{C}$ for $5 \mathrm{~min}$ and then cooled to $0{ }^{\circ} \mathrm{C}$. A freshly prepared solution of benzoic anhydride $\left(0.044 \mathrm{M}\right.$ in $\left.\mathrm{CH}_{2} \mathrm{Cl}_{2}, 0.84 \mathrm{~mL}, 37 \mu \mathrm{mol}\right)$ was added over a period of 10 $\mathrm{min}$. The reaction mixture was stirred at $0{ }^{\circ} \mathrm{C}$ for $2 \mathrm{~h}$, warmed up to $\mathrm{rt}$, and stirred for an additional $2 \mathrm{~h}$. The reaction mixture was quenched with saturated aqueous $\mathrm{NaHCO}_{3}(10 \mathrm{~mL})$ and extracted with $\mathrm{CH}_{2} \mathrm{Cl}_{2}(3 \times 10 \mathrm{~mL})$. The combined organic extracts were dried over $\mathrm{Na}_{2} \mathrm{SO}_{4}$, filtered through Celite, and the solvent was removed in vacuo to yield crude 33 . Purification by silica gel chromatography (10 to $25 \%$ EtOAc in hexanes) gave $15.5 \mathrm{mg}(56 \%$ ) of $\mathbf{3 3}$ as a colorless oil: IR (neat) 3434, 2910, 2848, 1719, 1510, 1451, 1272, 1243, 1200, 1104, $1066,1024,860 \mathrm{~cm}^{-1 ; 1} \mathrm{H}$ NMR $\left(\mathrm{CDCl}_{3}, 400 \mathrm{MHz}\right) \delta 8.10(\mathrm{~d}, J=8.4 \mathrm{~Hz}, 2 \mathrm{H}), 7.65(\mathrm{t}, J=7.6$ $\mathrm{Hz}, 1 \mathrm{H}), 7.53(\mathrm{t}, J=7.6 \mathrm{~Hz}, 2 \mathrm{H}), 7.41(\mathrm{t}, J=7.6 \mathrm{~Hz}, 2 \mathrm{H}), 7.26(\mathrm{~m}, 9 \mathrm{H}), 6.70(\mathrm{~d}, J=8.8 \mathrm{~Hz}$, $2 \mathrm{H}), 5.59(\mathrm{~s}, 1 \mathrm{H}), 5.41(\mathrm{~d}, J=1.6 \mathrm{~Hz}, 1 \mathrm{H}), 5.37(\mathrm{~m}, 2 \mathrm{H}), 5.26(\mathrm{~s}, 1 \mathrm{H}), 4.58(\mathrm{bd}, J=10.4 \mathrm{~Hz}$, $1 \mathrm{H}), 4.51(\mathrm{~s}, 2 \mathrm{H}), 4.08(\mathrm{dq}, J=4.4,8.8 \mathrm{~Hz}, 2 \mathrm{H}), 3.96(\mathrm{bs}, 1 \mathrm{H}), 3.72(\mathrm{~s}, 4 \mathrm{H}), 3.14(\mathrm{~m}, 3 \mathrm{H}), 2.84$ $(\mathrm{m}, 2 \mathrm{H}), 2.53(\mathrm{t}, J=8.4 \mathrm{~Hz}, 3 \mathrm{H}), 2.40(\mathrm{~d}, J=7.2,6.8 \mathrm{~Hz}, 1 \mathrm{H}), 2.36(\mathrm{t}, J=9.6 \mathrm{~Hz}, 1 \mathrm{H}), 1.80$ $(\mathrm{m}, 1 \mathrm{H}) ;{ }^{13} \mathrm{C} \mathrm{NMR}\left(\mathrm{CDCl}_{3}, 100 \mathrm{MHz}\right) \delta 166.8,158.6,142.1,138.3,133.8,133.7,130.5,130.4$, 129.9, 129.8, 129.7, 128.8, 128.2, 128.6, 128.0, 127.8, 125.8, 122.1, 118.0, 117.3, 114.3, 75.1, 74.3, 72.4, 71.0, 62.4, 60.4, 55.4, 41.2, 39.0, 37.3, 33.0, 29.9; HRMS $(\mathrm{M}+\mathrm{H})^{+}$calcd for $\mathrm{C}_{42} \mathrm{H}_{44} \mathrm{BrNO}_{7}+\mathrm{H}: 754.2379$, found 754.2385.

$\left(2 R^{\star}, 4 S^{\star}, 5 S^{\star}, 6 S^{\star}\right)$-4-Benzoyloxy-2-(2-benzyloxymethylallyl)-2-(3-bromobut-3-enyl)-5methanesulfonyloxy-6-(4-methoxybenzyl)piperidine-1-carboxylic acid phenyl ester (34)

A solution of $\mathbf{3 3}(20.0 \mathrm{mg}, 26.0 \mu \mathrm{mol})$ in $\mathrm{CH}_{2} \mathrm{Cl}_{2}(1.0 \mathrm{~mL})$ was cooled to $0{ }^{\circ} \mathrm{C}$. $\mathrm{Et}_{3} \mathrm{~N}(4.0 \mu \mathrm{l}$, $29 \mu \mathrm{mol})$ was added dropwise, followed by a catalytic amount of DMAP. The reaction mixture was stirred at $0{ }^{\circ} \mathrm{C}$ for $10 \mathrm{~min}$. A freshly prepared solution of $p$-methanesulfonyl chloride $(0.253$ $\mathrm{M}$ in $\mathrm{CH}_{2} \mathrm{Cl}_{2}, 0.15 \mathrm{~mL}, 40 \mu \mathrm{mol}$ ) was added over a period of $10 \mathrm{~min}$. The reaction mixture was stirred at $0{ }^{\circ} \mathrm{C}$ for $2 \mathrm{~h}$, warmed to $20^{\circ} \mathrm{C}$, and stirred for an additional $4 \mathrm{~h}$. The reaction mixture was quenched with saturated aqueous $\mathrm{NaHCO}_{3}(10 \mathrm{~mL})$ and extracted with $\mathrm{CH}_{2} \mathrm{Cl}_{2}$ $(3 \times 10 \mathrm{~mL})$. The combined organic extracts were dried over $\mathrm{Na}_{2} \mathrm{SO}_{4}$, filtered through Celite, and the solvent was removed in vacuo to yield crude 34 . Purification by silica gel chromatography ( 10 to $25 \%$ EtOAc in hexanes) gave $21.0 \mathrm{mg}$ ( $97 \%$ ) of $\mathbf{3 4}$ as a colorless oil: IR (neat) 2923, 1722, 1513, 1450, 1344, 1268, 1177, 1032, $949 \mathrm{~cm}^{-1 ; 1} \mathrm{H} \mathrm{NMR}\left(\mathrm{CDCl}_{3}, 400\right.$ MHz) $\delta 8.10(\mathrm{dd}, J=0.8,8.0 \mathrm{~Hz}, 2 \mathrm{H}), 7.66(\mathrm{dt}, J=1.2,7.2 \mathrm{~Hz}, 1 \mathrm{H}), 7.54(\mathrm{t}, J=8.4 \mathrm{~Hz}, 2 \mathrm{H})$, $7.41(\mathrm{t}, J=7.2 \mathrm{~Hz}, 2 \mathrm{H}), 7.26(\mathrm{~m}, 9 \mathrm{H}), 6.79(\mathrm{~d}, J=8.8 \mathrm{~Hz}, 2 \mathrm{H}), 5.65(\mathrm{t}, J=8.4 \mathrm{~Hz}, 1 \mathrm{H}), 5.58$ (d, $J=1.2 \mathrm{~Hz}, 1 \mathrm{H}), 5.41(\mathrm{~d}, J=2.0 \mathrm{~Hz}, 2 \mathrm{H}), 5.33(\mathrm{bs}, 1 \mathrm{H}), 4.91(\mathrm{~m}, 1 \mathrm{H}), 4.88(\mathrm{~s}, 1 \mathrm{H}), 4.52$ (s, 2H), $4.14(\mathrm{dd}, J=12.8,40.4 \mathrm{~Hz}, 2 \mathrm{H}), 3.77(\mathrm{~s}, 3 \mathrm{H}), 3.24(\mathrm{~m}, 2 \mathrm{H}), 2.92(\mathrm{~m}, 3 \mathrm{H}), 2.77(\mathrm{~s}, 3 \mathrm{H})$, $2.53(\mathrm{~m}, 3 \mathrm{H}), 2.37(\mathrm{t}, J=11.6 \mathrm{~Hz}, 1 \mathrm{H}), 1.80(\mathrm{~m}, 1 \mathrm{H}) ;{ }^{13} \mathrm{C} \mathrm{NMR}\left(\mathrm{CDCl}_{3}, 100 \mathrm{MHz}\right) \delta 165.5$, 159.0, 151.0, 141.6, 138.3, 133.9, 133.3, 130.48, 130.0, 129.7, 129.4, 128.9, 128.6, 128.5, 128.0, 127.8, 126.0, 122.1, 118.8, 118.7, 117.4, 114.6, 78.9, 74.5, 72.41, 71.2, 61.1, 60.4, 55.4, 40.7, 38.6, 37.3, 33.2, 32.1, 29.9; HRMS $(\mathrm{M}+\mathrm{H})^{+}$calcd for $\mathrm{C}_{43} \mathrm{H}_{46} \mathrm{BrNO}_{9} \mathrm{~S}+\mathrm{H}: 832.2155$, found 832.2145 . 
$\left(2 S^{\star}, 4 R^{\star}, 6 S^{\star}, 7 R^{\star}\right)$-4-(2-Benzyloxymethylallyl)-4-(3-bromobut-3-enyl)-2-(4-methoxybenzyl)-7oxa-3-aza-bicyclo[4.1.0]heptane-3-carboxylic acid phenyl ester (23)

A solution of $34(14.0 \mathrm{mg}, 16.8 \mu \mathrm{mol})$ in $\mathrm{MeOH}(1.0 \mathrm{~mL})$ was cooled to $0{ }^{\circ} \mathrm{C}$. Solid $\mathrm{NaOH}$ (4.7 $\mathrm{mg}, 0.12 \mathrm{mmol}$ ) was added in one portion. The reaction mixture was stirred at $0{ }^{\circ} \mathrm{C}$ for 30 min, warmed up to rt, and stirred for an additional $10 \mathrm{~min}$. Solid $\mathrm{K}_{2} \mathrm{CO}_{3}(100 \mathrm{mg})$ was added followed by $\mathrm{CH}_{2} \mathrm{Cl}_{2}(15 \mathrm{~mL})$. The resulting heterogeneous mixture was filtered through Celite, and the solvent was removed in vacuo to yield crude $\mathbf{2 3}$. Purification by silica gel chromatography (5 to $15 \%$ EtOAc in hexanes) gave $10 \mathrm{mg}(94 \%)$ of $\mathbf{2 3}$ as a colorless oil: IR (neat) 2923, 2855, 1713, 1515, 1456, 1379, 1250, $1203 \mathrm{~cm}^{-1 ;}{ }_{1} \mathrm{H} \mathrm{NMR}\left(\mathrm{CDCl}_{3}, 400 \mathrm{MHz}\right) \delta$ $7.39(\mathrm{t}, J=7.6 \mathrm{~Hz}, 1 \mathrm{H}), 7.30(\mathrm{~m}, 9 \mathrm{H}), 7.12(\mathrm{~d}, J=7.2 \mathrm{~Hz}, 2 \mathrm{H}), 6.83(\mathrm{~d}, J=8.8 \mathrm{~Hz}, 2 \mathrm{H}), 5.58$ $(\mathrm{s}, 1 \mathrm{H}), 5.39(\mathrm{~d}, J=2.0 \mathrm{~Hz}, 1 \mathrm{H}), 5.32(\mathrm{~d}, J=1.2 \mathrm{~Hz}, 1 \mathrm{H}), 5.10(\mathrm{~s}, 1 \mathrm{H}), 4.78(\mathrm{~m}, 1 \mathrm{H}), 4.49(\mathrm{dd}$, $J=18.4,5.2 \mathrm{~Hz}, 2 \mathrm{H}), 3.98(\mathrm{q}, J=12.8 \mathrm{~Hz}, 2 \mathrm{H}), 3.80(\mathrm{~s}, 3 \mathrm{H}) 3.38(\mathrm{~m}, 1 \mathrm{H}), 3.09(\mathrm{dq}, J=4.4$, $2.8 \mathrm{~Hz}, 1 \mathrm{H}), 2.93(\mathrm{~d}, J=13.6 \mathrm{~Hz}, 1 \mathrm{H}), 2.87(\mathrm{dd}, J=3.2,12.0 \mathrm{~Hz}, 1 \mathrm{H}), 2.75(\mathrm{~m}, 2 \mathrm{H}), 2.58(\mathrm{~d}$, $J=13.6 \mathrm{~Hz}, 1 \mathrm{H}), 2.37(\mathrm{~m}, 3 \mathrm{H}), 1.80(\mathrm{dd}, J=4.0,4.4 \mathrm{~Hz}, 1 \mathrm{H}), 1.60(\mathrm{~m}, 1 \mathrm{H}) ;{ }^{13} \mathrm{C} \mathrm{NMR}$ $\left(\mathrm{CDCl}_{3}, 100 \mathrm{MHz}\right) \delta 158.5,151.1,142.8,138.1,133.8,130.7,130.1,129.7,129.7,128.7$, $128.1,128.0,125.9,122.1,117.4,117.2,114.1,73.6,72.6,59.9,55.9,55.4,49.3,48.8,42.3$, 39.0, 38.3, 37.1, 34.7 (29.9 r); HRMS (M+H) ${ }^{+}$calcd for $\mathrm{C}_{35} \mathrm{H}_{38} \mathrm{BrNO}_{5}+\mathrm{H}: 632.2012$, found 632.2033 .

\section{Supplementary Material}

Refer to Web version on PubMed Central for supplementary material.

\section{Acknowledgements}

We express appreciation to the National Institutes of Health (Grant GM 34442) for financial support of this research. D.G. also thanks the Burroughs-Wellcome Fellowship Fund for a second year Graduate Assistantship. The authors thank Dr. Sabapathy Sankar for NMR interpretation. NMR and mass spectra data were obtained at NCSU instrumentation laboratories, which were established by grants from the North Carolina Biotechnology Center and the National Science Foundation (Grants CHE-9509532 and CHE- 0078253).

\section{References}

1. Borel, JF., editor. Cyclosporin A. Elsevier Biochemical Press; Amsterdam: 1982.

2. Schreiber SL. Cell 1992;70:365. [PubMed: 1379518]

3. (a) Schreiber SL. Science 1991;251:283. [PubMed: 1702904] (b) Kannedy MS, Deeg HJ, Storb N, Thomas ED. Transplant Proc 1983;15:471.

4. Sakamoto K, Tsujii E, Abe F, Nakanishi T, Yamashita M, Shigematsu N, Izumi S, Okuhara M. J Antibiot 1996;46:37. [PubMed: 8609083]

5. For total syntheses of (-)-FR901483, see: (a) Snider BB, Lin H. J Am Chem Soc 1999;121:7778. (b) Scheffler G, Seike H, Sorensen EJ. Angew Chem, Int Ed 2000;39:4593. (c) Ousmer M, Braun NA, Ciufolini MA. Org Lett 2001;3:765. [PubMed: 11259057]. For the total syntheses of (+)-FR901483, see: (d) Maeng JH, Funk RL. Org Lett 2001;3:1125. [PubMed: 11348175] (e) Kan T, Fujimoto T, Ieda S, Asoh Y, Kitaoka H, Fukujama T. Org Lett 2004;6:2729. [PubMed: 15281755] (f) Brummond KM, Hong SP. J Org Chem 2005;70:907. [PubMed: 15675848]. For approaches to FR901483 and its analogues, see: (g) Quirante J, Escolano C, Massot M, Bonjoch J. Tetrahedron 1997;53:1391. (h) Yamazaki N, Suzuki H, Kibajashi C. J Org Chem 1997;62:8280. [PubMed: 11671957] (i) Braun NA, Ciufolini MA, Peters K, Peters EM. Tetrahedron Lett 1998;39:4667. (j) Snider BB, Lin H, Foxman BM. J Org Chem 1998;63:6442. (k) Bonjoch J, Diaba F, Puigbo E, Sole D, Segarra V, Santamaria L, Beleta J, Ryder H, Palacios JM. Biorg Med Chem 1999;7:2891. (1) Brummond KM, Lu J. Org Lett 2001;3:1347. [PubMed: 11348231] (m) Wardrop DJ, Zhang W. Org Lett 2001;3:2353. [PubMed: 11463314] (n) Bonjoch J, Diaba F, Puigbo E, Sole D. Tetrahedron Lett 2003;44:8387. (o) Panchaud P, Ollivier C, Renaud P, Zigmantas S. J Org Chem 2004;69:2755. [PubMed: 15074924]

6. (a) Comins DL, Zhang YM, Joseph SP. Org Lett 1999;1:657. [PubMed: 10823197] (b) Comins DL, Joseph SP, Goehring RR. J Am Chem Soc 1994;116:4719. 
7. The numbering scheme adopted is based on the accepted numbering for the natural product, (-)FR901483 ( compound 1, Scheme 1).

8. Comins DL, Brown JD. Tetrahedron Lett 1986;27:4549.

9. Larock RC, Hightower TR, Kraus GA, Hahn P, Zheng D. Tetrahedron Lett 1995;36:2423.

10. Comins DL, Stoltze DA, Thakker P, McArdle CL. Tetrahedron Lett 1998;39:5693.

11. For reviews on $A^{(1,3)}$ strain, see: (a) Hoffmann RW. Chem Rev 1989;89:1841. (b) Johnson F. Chem Rev 1968;68:375.

12. Kajiro H, Mitamura S, Mori A, Hiyama T. Tetrahedron Lett 1999;40:1689.

13. A variety of basic hydrolysis conditions $\left(\mathrm{K}_{2} \mathrm{CO}_{3}, \mathrm{MeOH} ; \mathrm{KCN}, \mathrm{MeOH}\right)$ resulted in acetate hydrolysis together with carbamate removal or significant decomposition (DBU, $\mathrm{MeOH} ; \mathrm{NH}_{3}, \mathrm{MeOH}$ ). Other acidic type hydrolysis conditions $\left(10 \%\right.$ aqueous $\mathrm{HCl}, \mathrm{EtOH} ; 10 \%$ aqueous $\mathrm{H}_{2} \mathrm{SO}_{4}, \mathrm{EtOH}$; $\left.\mathrm{HBF}_{4} \cdot \mathrm{OEt}_{2}, \mathrm{MeOH}\right)$ resulted in significant polymerization upon scale up.

14. Acetal exchange was necessary as subjection of the (1,3)-dioxolane acetal protected version of compound 16 to the Sakurai conjugate allylation conditions (allyl silane 17, $\mathrm{TiCl}_{4}, \mathrm{CH}_{2} \mathrm{Cl}_{2}$ ) resulted in the Lewis acid coordinating to the acetal moiety leading to attack at the side chain.

15. (a) Trost BM, Chan DMT, Nanninga TN. Org Synth 1984;62:58. (b) Marko IE, Plancher JM. Tetrahedron Lett 1999;40:5259. (c) Dumeunier R, Markó IE. Tetrahedron Lett 2000;41:10219.

16. Sakurai H, Hosomi A, Hayashi J. Org Synth 1984;62:86.

17. (a) Evans DA, Rajapakse HA, Stenkamp D. Angew Chem Int Ed 2002;41:4569. (b) Kelly DR, Mahdi JG. Tetrahedron Lett 2002;43:511.

18. Hagen G, Mayr H. J Am Chem Soc 1991;113:4954.

19. The stereochemistry of the major diastereomer 19 is believed to be as depicted based on analogy with the allylation reaction in 25 yielding the structurally similar compound 31 with unambiguously established stereochemical correlation of the substituents on the piperidone ring (See Scheme 8).

20. (a) Comins DL, LaMunyon DH, Chen X. J Org Chem 1997;62:8182. [PubMed: 11671929] (b) Kuethe JT, Comins DL. Org Lett 1999;1:1031. [PubMed: 10825955]. For discussions on stereoelectronic control in reactions of this type, see: (c)DeslongchampsPStereoelectronic Effects in Organic ChemistryPergamonNew York1983Chapter 6. (d) Stevens RV. Acc Chem Res 1984;17:289.

21. For a few examples of conjugate allylations in dihydropyridones unsubstituted $\alpha$ to the nitrogen at the vinylogous amide portion of the molecule, see: (a) Sato M, Aoyagi S, Yago S, Kibayashi C. Tetrahedron Lett 1996;37:9063. (b) Comins DL, Killpack MO, Despagnet E, Zeller E. Heterocycles 2002;58:505. (c) Kranke B, Hebrault D, Schultz-Kukula M, Kunz H. Syn Lett 2004;4:671.

22. (a) See Table 1 in Supporting Information. (b) See Table 2 in Supporting Information.

23. MacSpartan Pro minimization employing MMFF conformer distribution of ketone 19 revealed that the compound existed in a twist-boat conformation with no apparent thermodynamic bias for the desired epimerization to take place.

24. We could epimerize the C-7 acetate functionality $15 \mathrm{a}$, by prolonged exposure to acetic acid, and prepare the C-7 epimer of 16 . However, all our attempts at the conjugate allylation reaction to install the $\mathrm{C}-1$ quaternary center failed, presumably due to the fact that the bulky TBS ether (which is now epimeric at C-7 in dihydropyridone 16) blocks the $\alpha$-face during the addition step. Since this is the face the nucleophile has to approach in order to maintain a low energy, chair-like transition state, attack does not occur.

25. White JD, Hanselmann R, Wardrop DJ. J Am Chem Soc 1999;121:1106.

26. (a) Dobler MR, Bruce I, Cederbaum F, Cooke NG, Diozario LJ, Hall RG, Irving E. Tetrahedron Lett 2001;42:8281. (b) Takahara PM, Freudenberger JH, Konradi AW, Pedersen SF. Tetrahedron Lett 1989;30:7177. (c) Matsamuto T, Yamaguchi H, Tanabe M, Yasui Y, Suzuki K. Tetrahedron Lett 2000;41:8393. (d) Kawatsura M, Kishi E, Kito M, Sakai H, Shirahama H, Matsuda F. Synlett 1997;55:479. (e) Molander GA, Kenny C. J Org Chem 1988;53:2134. (f) Fleming M, McMurry JJ. Org Synth 1982;60:113. (g) McMurry J. Chem Rev 1989;89:1513. (h) Molander GA, Harris CR. Tetrahedron 1998;54:3321.

27. (a) Crimmins MT, Gould LD. J Am Chem Soc 1987;109:6199. (b) Rossi R, Carpita A, Ciofalo M, Lippolis V. Tetrahedron 1991;47:8443.

28. Nicolaou KC, Gray DLF, Montagnon T, Harrison ST. Angew Chem Int Ed 2002;41:996. 
29. During the course of the oxidation of 27 to 28 , we observed a small amount (15\%) of the piperidone product resulting from hydrolysis of the silyl enol ether functionality.

30. During the course of the oxidation of 27 to 28 we were surprised to see that exposure to our earlier catalytic oxidation conditions ( $\mathrm{Pd}(\mathrm{OAc})_{2}$ ( 0.1 equiv), $\mathrm{CuCl}$ ( 1 equiv), $\mathrm{O}_{2}, \mathrm{CH}_{3} \mathrm{CN}, 65^{\circ} \mathrm{C}$ ) gave only the undesired piperidone byproduct resulting from the hydrolysis of the silyl enol ether moiety.

31. (a) White JD, Blakemore PR, Browder CC, Hong J, Lincoln CM, Nagornyy PA, Robarge LA, Wardrop DL. J Am Chem Soc 2001;123:8593. [PubMed: 11525667] (b) Hara S, Dojo H, Takinami S, Suzuki A. Tetrahedron Lett 1983;24:731.

32. The product showed a small coupling constant $\left(J_{\mathrm{H} 6-\mathrm{H} 7}=2.0 \mathrm{~Hz}\right)$ in its ${ }^{1} \mathrm{H}-\mathrm{NMR}$, consistent with its trans-stereochemistry.

33. In a previous experiment we had discovered that exposure of diol 32 to $\mathrm{MsCl}$ (1.0 equiv) in the presence of $\mathrm{Et}_{3} \mathrm{~N}$ and DMAP resulted in the formation of the $\mathrm{C}-7$ mesylate as the major compound of a 3:1 mixture of C-8/C-7 regioisomers. Thus, in order to obtain the desired epoxide 23 we needed to selectively derivatize the $\mathrm{C}-8$ hydroxyl first. This led to the $\mathrm{C}-8$ benzoate ester formation/C-7 mesylation route.

34. Mashimo K, Sato Y. Tetrahedron 1970;26:803.

35. (a) Gauthier DR Jr, Bender SL. Tetrahedron Lett 1996;37:13. (b) Hudlickly T, Rinner U, Gonzalez D, Akgun H, Schilling S, Siengalewicz P, Martinot TA, Pettit GR. J Org Chem 2002;67:8726. [PubMed: 12467383] 


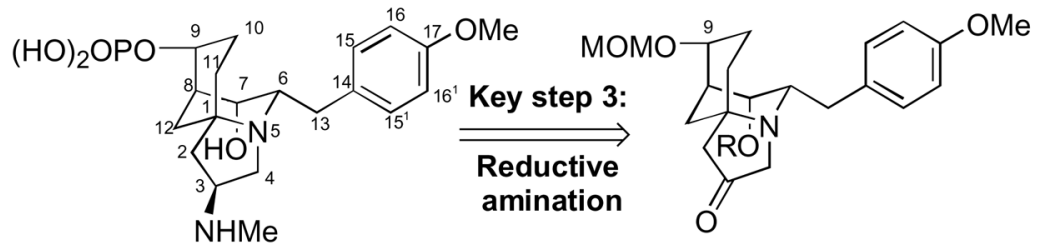
(-)-FR901483 (1)
2, $R=T B S$

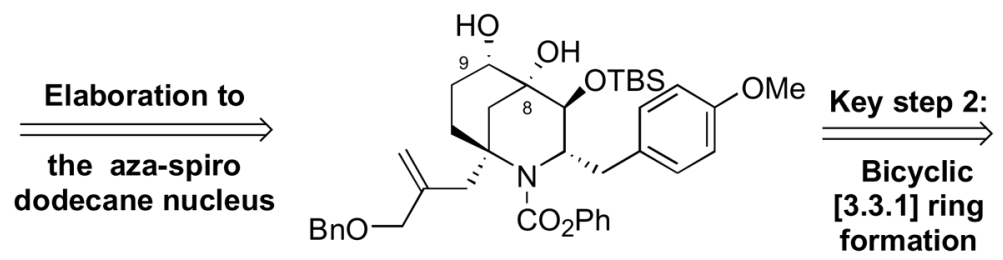

3

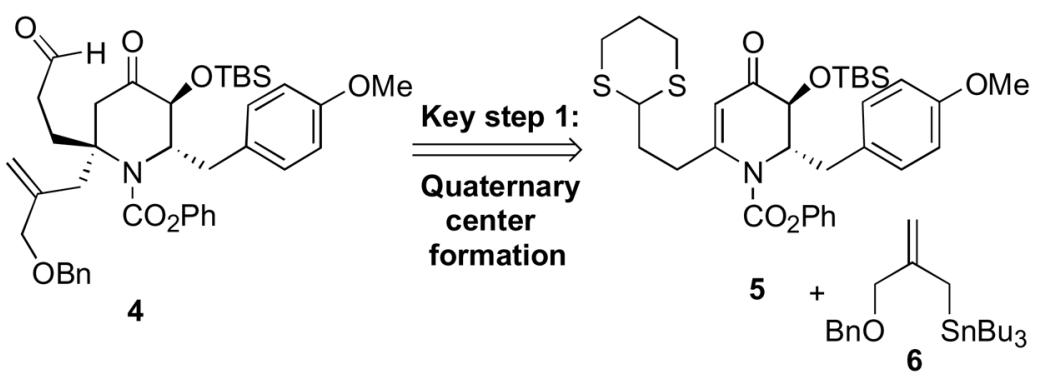

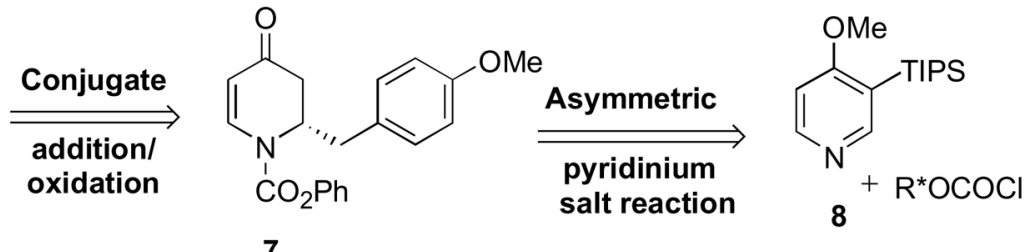

Scheme 1.

First Generation Retrosynthetic Analysis of (-)-FR901483 


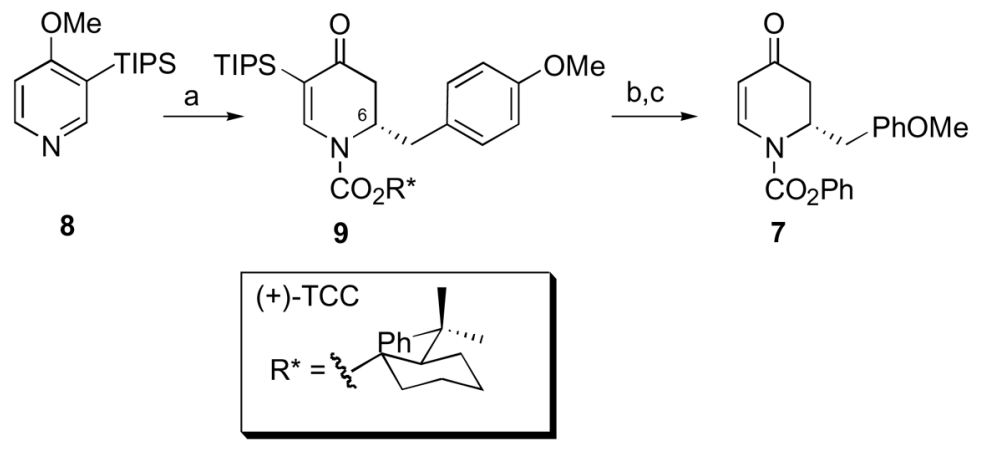

\begin{abstract}
${ }^{a}$ Reaction conditions: (a) (+)-TCC chloroformate, $-78{ }^{\circ} \mathrm{C}$; then $\mathrm{p}-\mathrm{OMeBnMgCl}$; then $\mathrm{H}_{3} \mathrm{O}^{+}$(89\%, $\left.d r=95: 5\right)$; (b) $\mathrm{NaOMe}, \mathrm{MeOH}$; then $\mathrm{H}_{3} \mathrm{O}^{+}$(90\%); (c) $n-\mathrm{BuLi}$, THF; then $\mathrm{PhOCOCl}(98 \%)$.
\end{abstract}

Scheme 2.

Synthesis of Dihydropyridone $6^{\mathrm{a}}$ 
<smiles>COC1=CC(CCC2OCCO2)N(C(=O)OCc2ccccc2)C(CPc2ccccc2)C1</smiles>

10

11

12

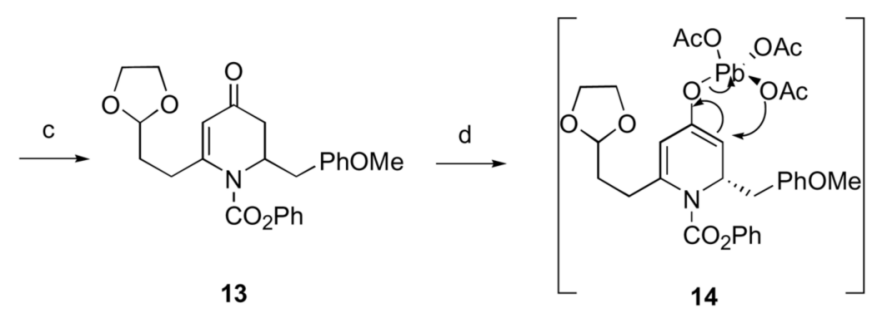<smiles>COC(=O)n1c(CCC2OCCO2)cc(=O)c(OC)c1Cc1ccccc1</smiles><smiles>COC(=O)c1ccccc1</smiles>

$15 a$
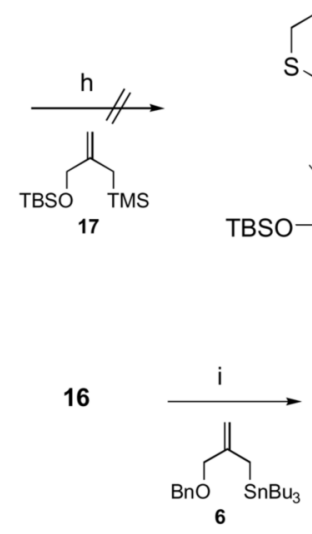<smiles>C=C(COC)C[C@]1(CCC2SCCCS2)CC(=O)C(O[Sb])[C@H](Cc2ccccc2)N1C(=O)Oc1ccccc1</smiles>

16

16

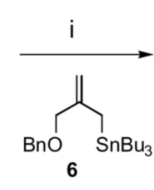<smiles>C=C(CO)C[C@]1(CCC2SCC[C@H]2C)CC(=O)C([OH2+])[C@H](Cc2ccccc2)N1C(=O)Oc1ccccc1</smiles>

19

${ }^{\mathrm{a}}$ Reaction conditions: (a) $\mathrm{PhOCOCl},-78{ }^{\circ} \mathrm{C}$; then $\mathrm{p}-\mathrm{MeOBnMgCl}$; then $\mathrm{H}_{3} \mathrm{O}^{+}(91 \%)$; (b) $\mathrm{CuBr} . \mathrm{SMe}_{2}, \mathrm{TMSCl}, \mathrm{Et}_{3} \mathrm{~N}, \quad(2$-[1,3]dioxolan-2-yl-ethyl)magnesium bromide (used crude); (c) $\mathrm{Pd}(\mathrm{OAc})_{2}$ (0.1 equiv), $\mathrm{CuCl}$ (1 equiv), $\mathrm{O}_{2}, \mathrm{CH}_{3} \mathrm{CN}, 65{ }^{\circ} \mathrm{C}$ (70\%, 2 steps); (d) $\mathrm{Pb}(\mathrm{OAc})_{4}$, toluene, reflux (75\%, $d r=15: 1$ ); (e) $\mathrm{ScOTf}_{3}$ (0.2 equiv), $\mathrm{H}_{2} \mathrm{O}: \mathrm{MeOH}, 1: 4$ (100\%); (f) TBSCl, imidazole (90\%); (g) $\mathrm{BF}_{3} \cdot \mathrm{OEt}_{2}, \mathrm{HS}\left(\mathrm{CH}_{2}\right)_{3} \mathrm{SH}$ (95\%); (h) $\mathrm{TiCl}_{4}$, $\mathrm{CH}_{2} \mathrm{Cl}_{2}$. (i) TMSOTf, $\mathrm{CH}_{2} \mathrm{Cl}_{2}(65 \%, d r=5.5: 1)$.

Scheme 3.

C-1 Quaternary Center Installation Via Conjugate Allylation ${ }^{\mathrm{a}}$ 


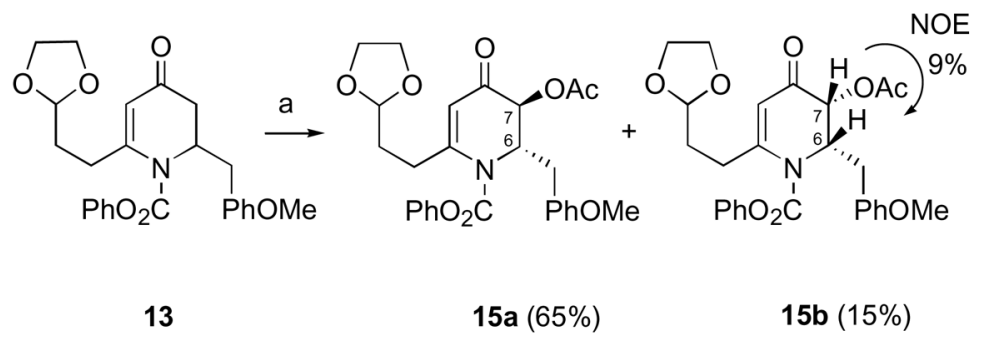

${ }^{a}$ Reaction conditions: (a) $\mathrm{Pb}(\mathrm{OAc})_{4}, \mathrm{AcOH}$ ( $5 \mathrm{~mol} \%$ ), toluene, reflux, 3 days.

Scheme 4.

Confirmation of the C-7 Stereochemistry ${ }^{\mathrm{a}}$ 

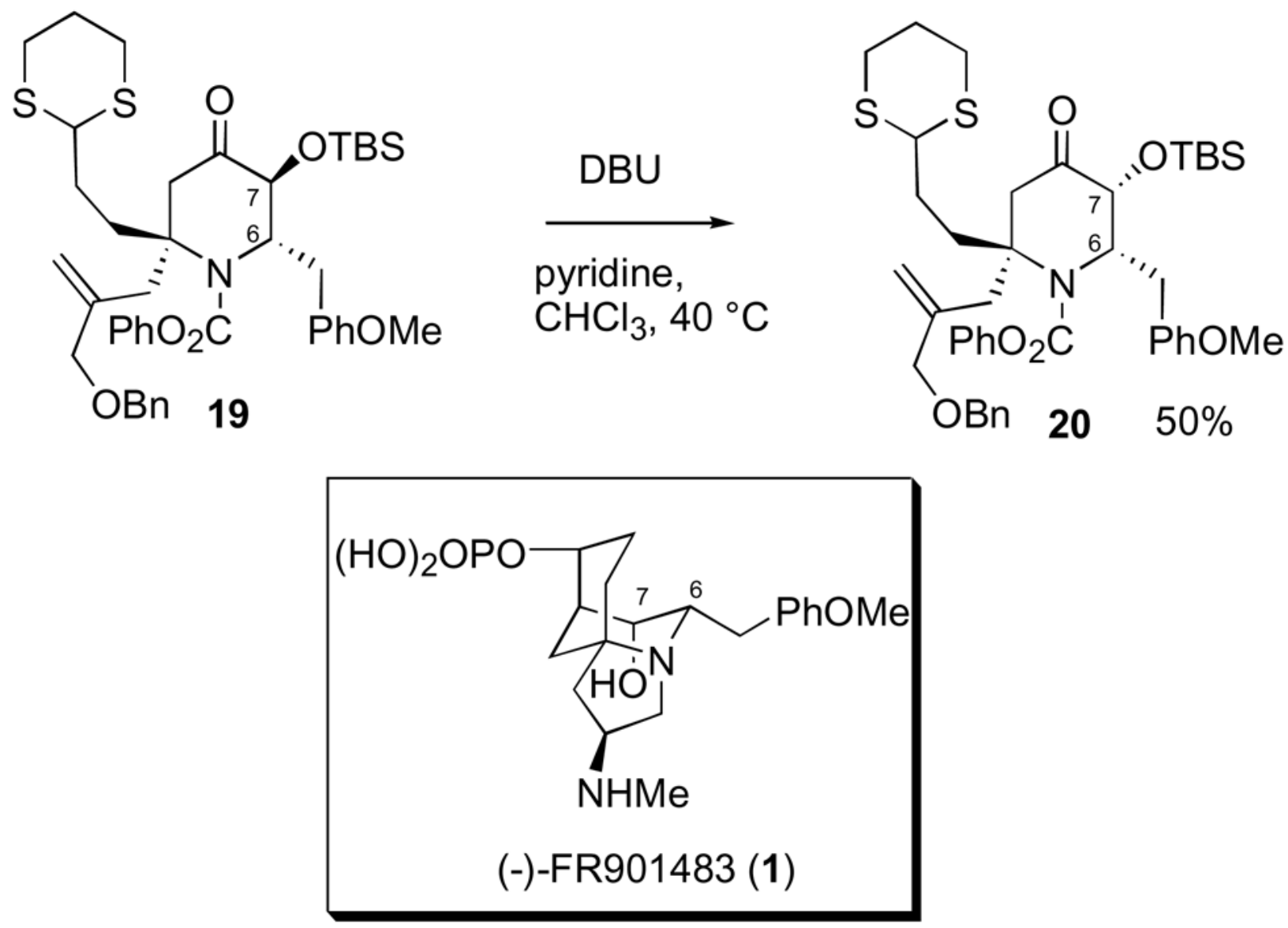

Scheme 5.

Epimerization of the Silyl Ether in Dihydropyridone 19 
<smiles>C=C(COc1ccccc1)C[C@]1(CCC2SCCCS2)CC(=O)[C@@H]([O-])[C@H](Cc2ccccc2)N1C(=O)Oc1ccccc1</smiles>

19<smiles>C=C(COc1ccccc1)C[C@]1(CCC=O)CC(=O)[C@@H]([OH2+])[C@H](Cc2ccccc2)N1C(=O)Oc1ccccc1</smiles>

21

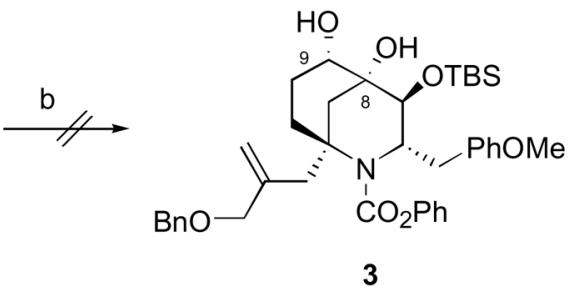

${ }^{a}$ Reaction conditions: (a) $\mathrm{CaCO}_{3}, \mathrm{Mel}, \mathrm{H}_{2} \mathrm{O}: \mathrm{CH}_{3} \mathrm{CN} \mathrm{1:9} \mathrm{(100 \% ,} \mathrm{crude);}$ (b) see Table 2.

Scheme 6.

Attempted Pinacol Coupling of Keto-Aldehyde 21 


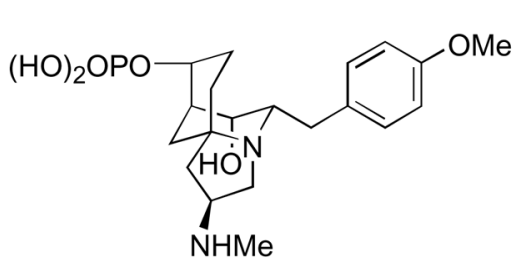

(-)-FR901483 (1)

\section{Key step 3: \\ Reductive amination}

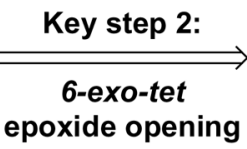<smiles>C=C(Br)CC[C@@]1(CC(=C)COc2ccccc2)C[C@@H]2O[C@H]2[C@H](Cc2ccccc2)N1C(=O)OC</smiles>

23

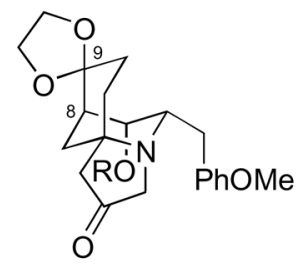

22, $\mathrm{R}=\mathrm{TBS}$
Key step 1:

Stereoselective epoxide formation

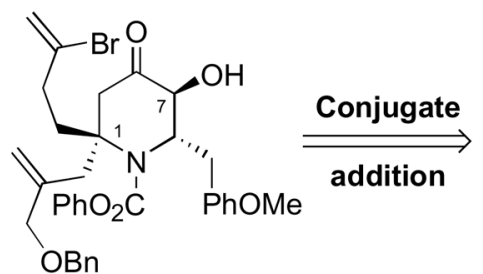

24<smiles>C=C(Br)CCC1=CC(=O)C([OH+][SbH2])[C@H](CPO)N1C(=O)Oc1ccccc1</smiles>

25

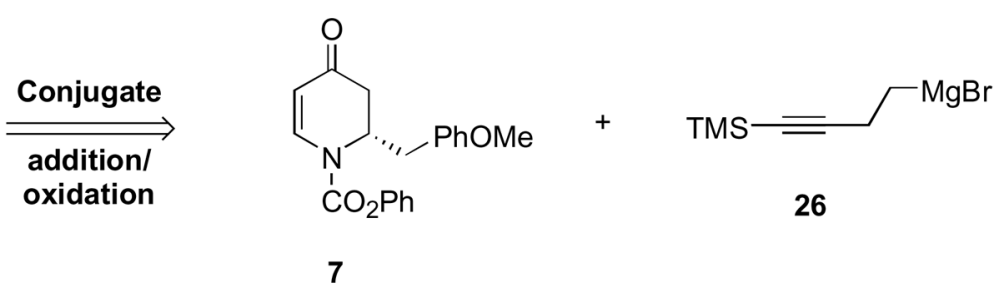

Scheme 7.

Second Generation Retrosynthetic Analysis 


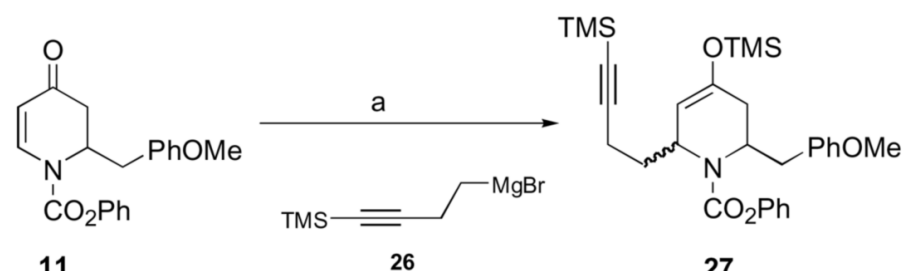

11

26

27<smiles>C=C(Br)CCC1=CC(=O)CC(Cc2ccccc2)N1C(=O)OCc1ccccc1</smiles>

28

29<smiles>C=C(Br)CCC1=CC(=O)[C@H]([OH+][Sb])[C@H](Cc2ccccc2)N1C(=O)OC</smiles>

25

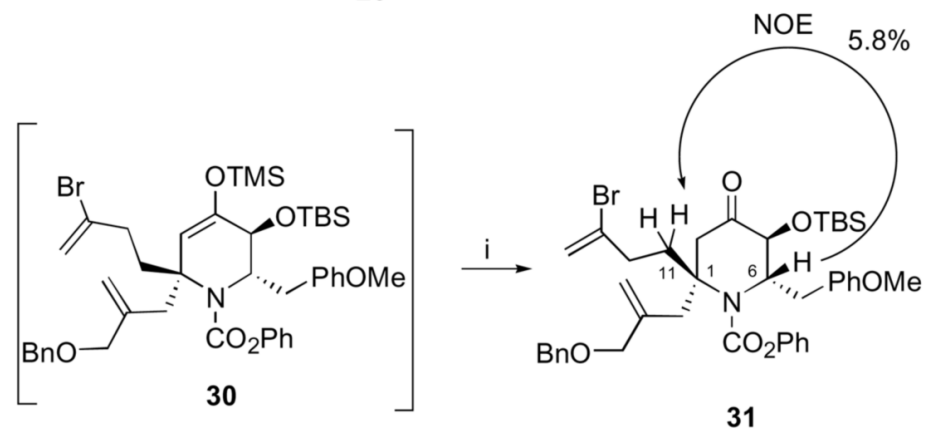

${ }^{\mathrm{a}}$ Reaction conditions: (a) $\mathrm{CuBr} \cdot \mathrm{SMe}_{2}, \mathrm{TMSCl} \mathrm{Et}_{3} \mathrm{~N}, 26$ (used crude); (b) IBX (1.2 equiv), 4-methoxypyridine $\mathrm{N}$-oxide. $\mathrm{H}_{2} \mathrm{O}$ (1.2 equiv), DMSO (55\%, 2 steps); (c) TBAF, THF, $-40{ }^{\circ} \mathrm{C}$ (93\%); (d) $B-9-$ $\mathrm{BBN}, \mathrm{CH}_{2} \mathrm{Cl}_{2}$ (85\%); (e) $\mathrm{Pb}(\mathrm{OAc})_{4}$, toluene, reflux (69\%, $\left.d r=15: 1\right)$; (f) $\mathrm{ScOTf}_{3}$ (0.2 equiv), $\mathrm{H}_{2} \mathrm{O}: \mathrm{MeOH} 1: 4$ (100\%); (g) TBSCl, imidazole (90\%); (h) TMSOTf, 6, $\mathrm{CH}_{2} \mathrm{Cl}_{2}$ (crude); (i) silica gel (50 equiv), oxalic acid (0.1 equiv), $\mathrm{MeOH}: \mathrm{H}_{2} \mathrm{O}$ 10:1 (85\%, 2 steps, $\left.d r>99: 1\right)$.

Scheme 8.

C-1 Quaternary Center Installation in Piperidone $31^{\mathrm{a}}$ 
<smiles>[B-]O[C@@H]1C(=O)C[C@](CCC(=C)Br)(CC(=C)COc2ccccc2)N(C(=O)Oc2ccccc2)[C@H]1CP</smiles>

31<smiles>C=C(Br)CC[C@@]1(CC(=C)COc2ccccc2)C[C@@H](O)[C@H](O)[C@H]1CPc1ccccc1</smiles>

32<smiles>C=C(Br)CC[C@]1(CC(=C)COc2ccccc2)C[C@H](OC(C)(C)C)C(O)[C@H](CPc2ccccc2)N1C(=O)OCc1ccccc1</smiles>

33<smiles>C=C(COc1ccccc1)C[C@@]1(CCC(Br)=CC)C[C@H](OC(C)(C)C)C(OC)[C@H](CPc2ccccc2)N1C(=O)OCc1ccccc1</smiles>

34

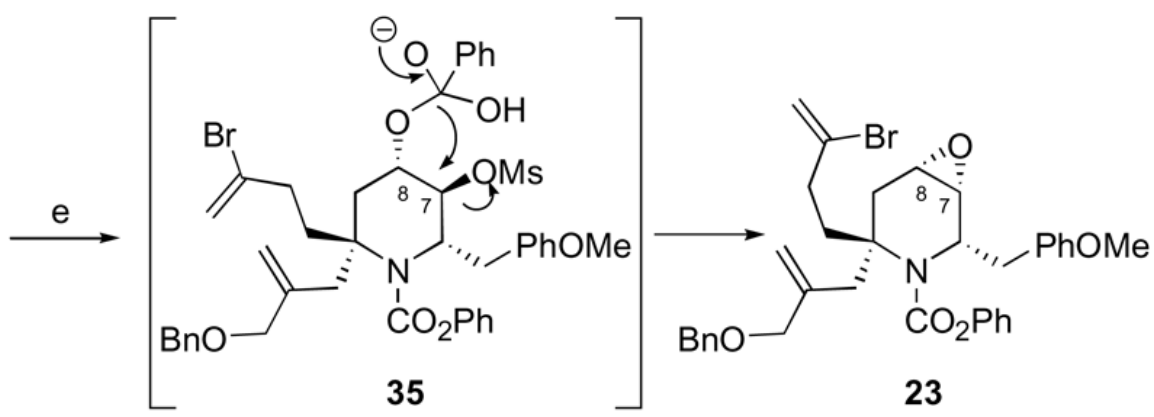

${ }^{a}$ Reaction conditions: (a) TBAF (1.2 equiv), THF, $-40{ }^{\circ} \mathrm{C}$ (crude); (b) $\mathrm{Me}_{4} \mathrm{~N}^{+} \mathrm{BH}(\mathrm{OEt})_{3}, \mathrm{AcOH}(92 \%, 2 \text { steps); (c) ( } \mathrm{PhCO})_{2} \mathrm{O}$ (1.0 equiv) $\mathrm{Et}_{3} \mathrm{~N}(2.0$ equiv), DMAP (cat), $\mathrm{CH}_{2} \mathrm{Cl}_{2}, 0{ }^{\circ} \mathrm{C}$ (56\%); (d) $\mathrm{MsCl} \mathrm{Et}_{3} \mathrm{~N}$ (2.0 equiv), DMAP (cat), $\mathrm{CH}_{2} \mathrm{Cl}_{2}$ (97\%); (e) $\mathrm{NaOH}, \mathrm{MeOH}(94 \%)$.

Scheme 9.

Synthesis of Key Epoxide Intermediate 23a 


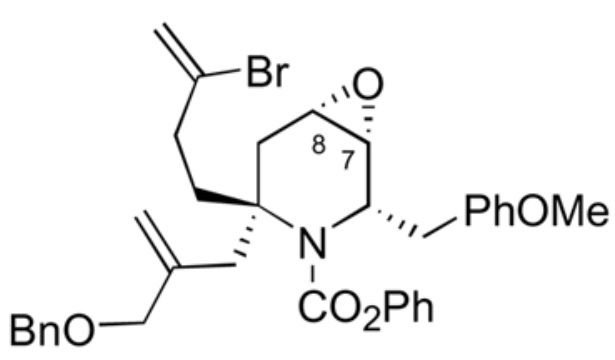

23

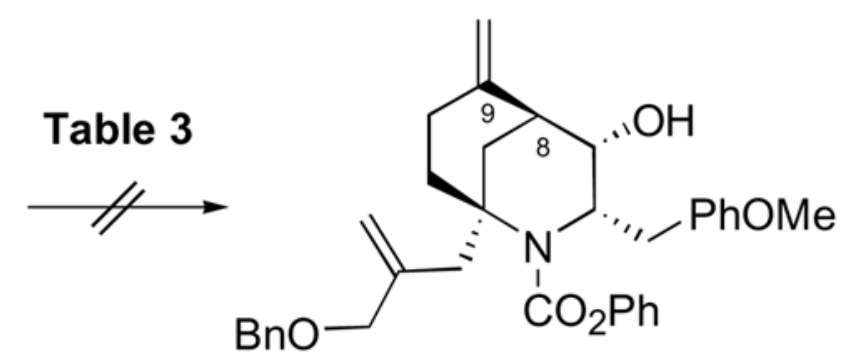

36

Scheme 10.

Attempted 6-exo-tet-Epoxide Opening in 23 